\title{
Global Dual Sourcing and Order Smoothing: The Impact of Capacity and Leadtimes
}

\author{
Robert N. Boute \\ Jan A. Van Mieghem \\ robert.boute@vlerick.com \\ vanmieghem@northwestern.edu
}

Nov. 20, 2012; Revised: Aug. 6, 2013; Dec. 12, 2013; Apr. 23, 2014

\begin{abstract}
After decades of offshoring production across the world, companies are rethinking their global networks. Local sourcing is receiving more attention, but it remains challenging to balance the offshore sourcing cost advantage against the increased inventories due to its longer leadtime, and against the cost and (volume-)flexibility of each source's capacity. To guide strategic allocation in this global network decision, this paper establishes reasonably simple prescriptions that capture the key drivers. We adopt a conventional discrete-time inventory model with a linear control rule that smoothes orders and allows an exact and analytically-tractable analysis of single and dual sourcing policies under normal demand. Distinguishing features of our model are that it captures each source's leadtime, capacity cost and flexibility to work overtime. We use Lagrange's inversion theorem to provide exact and simple square-root bound formulae for the strategic sourcing allocations and the value of dual sourcing. The formulae provide structural insight on the impact of financial, operational and demand parameters, and a starting point for quantitative decision making. We investigate the robustness of our results by comparing the smoothing policy with existing single and dual sourcing models in a simulation study that relaxes model assumptions.
\end{abstract}

\section{Introduction and Summary}

Over the past few decades it became conventional wisdom that factory jobs could be done cheaply in some far-flung corner of the world. The original impetus behind offshoring for Western firms was to reap savings from performing work in overseas countries with lower wages and materials costs. For several decades that strategy worked, often brilliantly, but now companies are rethinking their global networks. A special report in The Economist on Offshoring and Outsourcing (Jan. 19, 2013) reports the story of Lenovo, a Chinese technology group opening a US computer manufacturing line in North Carolina. The global labor "arbitrage" is running out: wages in China have nearly doubled since 2008, partly as a result of domestic minimum-wage policies (the country's 2011 fiveyear plan called for 13 percent average annual minimum-wage increases, a rate some provinces already exceeded (George et al. 2014)). The cost of shipping goods around the world has been rising sharply, and goods spend weeks in transit. Offshoring also often requires substantial safety inventory, the holding cost of which can outweigh the labor and materials cost advantage. Today, greater emphasis is placed on proximity to demand: responding to customers' new-product requests, 
shorter delivery times, and swift corrections to improve designs and quality has magnified the need for responsive and flexible supply chains. Local sourcing therefore is receiving increasing attention.

A complete reversal to local sourcing, however, may be unlikely and ill-advised. Indeed, the concepts of global and local sourcing are not mutually exclusive. Rather, the combined use of multiple supply sources, each of which is different and possesses unique advantages, might be better than any single sourcing strategy. Admittedly, multi-sourcing involves higher coordination costs, but a strategically configured portfolio of suppliers with complementary skills can often perform better than any individual supplier.

In this paper we analyze global sourcing for companies that have access to two sources with complementary competencies: a local source that is responsive but more expensive, and a global source that is (globally) more cost-efficient but with a longer leadtime Thile our policy can be used for two local sources, we adopt the setting where the low-cost source is offshore and far away from the local responsive source. Whereas the sourcing literature typically only considers sourcing and inventory related costs, we explicitly add each source's capacity cost and flexibility (modeled by its cost to work overtime). In the current economic climate of rising labor costs in some countries, and decreasing flexibility to go beyond regular working hours (driven by strong labor unions or by capacity rigidity due to high levels of automation), capacity cost and flexibility are increasingly relevant factors for sourcing decisions. The Economist (Jan. 19, 2013) mentions that one of the reasons why reshoring may less likely happen in Europe, compared to the US, is among others because Europe's labor markets are still fairly inflexible and costly. Labor flexibility still varies greatly from country to country. In a global economy where firms can go where they want, these differences have an effect.

Our approach assumes a wholly-owned global network where capacity costs and flexibility are directly relevant. We believe this approach provides a stepping stone to a decentralized system, where independent suppliers will in one way or another include a sourcing charge for capacity costs and flexibility, but a formal game-theoretic analysis is left for future research.

We adopt a conventional discrete-time inventory model with stochastic demand and a linear control rule that is capable to smooth orders to both sources. The reasons for analyzing this policy, which we will refer to as dual sourcing smoothing (DSS), are that smoothing policies are effective to reduce capacity requirements and are used in practice (see empirical evidence in next literature review) when companies face high labor or capital capacity costs. In addition, the linearity of smoothing policies provides analytic tractability with normally distributed demand and allows us to specify analytically the strategic sourcing allocations to both sources.

The optimality equations involve a polynomial of degree higher than the leadtime difference $L$ between the two sources. Given that a quartic is the polynomial of highest degree for which general finite analytic expressions for the roots can exist, we use Lagrange's inversion formula to solve the optimality equations for general $L$. Another technical contribution, for which we relied on Lagrange's technique, is the inclusion of general leadtimes for each source in a smoothing policy.

\footnotetext{
${ }^{1}$ Supply competencies can correspond to transportation modes so that our analysis also applies to balancing mixed-mode transportation or spot and forward market purchasing.
} 
To the best of our knowledge, the application of the Lagrange series to inventory theory appears novel.

The main contribution of this paper is to provide managerial guidelines for strategic sourcing (global, local or dual sourcing) based on exact formulae and simple square-root bounds that capture the impact of each source's cost, leadtime, capacity and flexibility for normally distributed demand. Specifically, we present a simple guideline that captures the tradeoff between these four parameters when deciding between local or global single sourcing with the standard base-stock policy. We show that single sourcing with order smoothing dominates using a base-stock policy in the presence of capacity costs and performs close to the optimal sourcing policy under capacity costs. We then extend order smoothing to dual sourcing and present formulae and bounds that specify the optimal volume fraction ordered from the global source (the strategic offshoring allocation), its corresponding total landed cost, and the value of dual sourcing (over single sourcing).

We show that order smoothing policies shine when dual sourcing faces capacity costs, inflexibility, or longer leadtime difference $L$ between both sources. The maximal value of dual sourcing smoothing then increases significantly and grows at the order of $L^{1 / 6}$. Moreover, the parameter region for which dual sourcing smoothing dominates capacitated single sourcing widens as $L$ increases. The square-root formulae that we present are sufficiently simple to provide a starting point for quantitative decision making to optimally trade-off cost and responsiveness. While simple, the formulae still capture the key parameters and thus provide structural insight on the impact of financial, operational and demand parameters on dual sourcing decisions.

Finally, a simulation study demonstrates the robustness of our results by relaxing the normality assumption and by comparing the policy with other policies studied in the literature.

\section{Related Literature}

Our work directly relates to two streams of research: dual sourcing inventory models and order smoothing policies. The dual-sourcing literature refers to inventory models where replenishment occurs through a regular channel and/or a more expensive, but faster expedited channel. The objective is to minimize the expected sum of procurement, holding and shortage costs over multiple periods. The dual-sourcing literature is very rich; we focus primarily on discrete review models. Fukuda (1964) shows that when the leadtime difference is one period, dual-base-stock policies are optimal. In a dual-base-stock policy, an expedited order is placed to bring the inventory position up to a first (expedite) base-stock level, after which a regular order is placed to bring it up to a second and higher (regular) base-stock level. Fukuda uses first-order conditions to derive expressions for the base-stock levels. Whittemore and Saunders (1977) extend Fukuda's (1964) model and show that when leadtimes differ by more than one period, the optimal policy is no longer a dual base-stock, but it depends on the entire ordering history and requires multidimensional dynamic programming.

Optimal dual sourcing policies are in general highly complex. Therefore, various heuristic policies are proposed in the literature. Veeraraghavan and Scheller-Wolf $(2008)$ introduce a dual-index dual-base-stock policy that tracks inventory positions over both regular and expedited leadtimes. 
Order-up-to levels for both inventory positions are computed using a simulation-based optimization procedure. The authors show that such a dual-index policy is nearly optimal when compared to state-dependent policies. Scheller-Wolf et al. (2006) consider single-index dual-base-stock policies, whose structure is identical to dual-index policies except that only one inventory position is tracked instead of two. The authors computationally show that its performance is comparable to the more complex dual-index policy. The single-index policy also allows closed form cost expressions under certain distributional assumptions. As such, this is the only other policy from which you can obtain insights by looking at the expressions. Sheopuri et al. (2010) generalize the class of dual-index policies. They show that the "lost sales inventory problem" is a special case of the dual sourcing problem and leverage this property to suggest new classes of policies with an order-up-to structure that perform equal to, or even slightly better than, the dual-index policy with the same computational requirements. One of their best performing policies is a base-stock policy for placing expedite orders and a Vector-base-stock policy for regular orders (this policy was inspired by Zipkin (2008) whose experiments showed that the Vector-base-stock policy outperforms the best base-stock policy for the lost sales inventory problem in a single source setting).

Rosenshine and Obee (1976) consider a standing order policy, which orders at a constant rate from the regular source and uses a base-stock policy for the emergency replenishment. Tagaras and Vlachos (2001) extend this policy to allow emergency replenishment within the regular review period. Allon and Van Mieghem (2010) refer to a standing order policy as a tailored base-surge (TBS) policy, where the regular source supplies the "base" demand and the fast source supplies the remaining "surge" demand using a base-stock policy. It is noteworthy that, by definition, a TBS policy is independent of the slow source's leadtime. This allows some mathematical tractability: Allon and Van Mieghem (2010) develop an analytical Brownian model that is asymptotically optimal for high sourcing volumes. Janakiraman et al. (2014) show that the TBS policy is optimal when demand comes from a two point distribution and when the probability of the smaller (base) demand is sufficiently large. They also show that TBS performance, relative to the optimal policy, improves as the lead time of the regular source increases.

Recently, the dual-sourcing literature is adopted in the context of global sourcing strategies, thus combining the advantages of global low-cost sourcing and local quick response manufacturing. Allon and Van Mieghem (2010) provide guidelines for determining the "strategic allocations," i.e., how the average total sourcing volume should be allocated to the global and local sources when the standing order or TBS policy is used. Wu and Zhang (2011) develop a game-theoretic model where multiple firms in a competitive setting may choose between efficient sourcing and responsive sourcing; a key feature of the game is that depending on the sourcing strategy, a firm may observe different signals about the uncertain market demand. Liu and Nagurney (2011) address the impact of demand and cost uncertainty in a supply chain network with offshoring and quick-response production. Using variational inequality theory, the authors formulate the governing equilibrium conditions of the competing manufacturers and a simulation study investigates the quantitative impact of demand and cost uncertainty. Recent empirical work by Jain et al. (2014) studies the 
impact of global sourcing and supplier diversification on inventory investment.

Our dual sourcing model also relates to the choice of mixed-mode transportation systems where a shipper can use two transport modes together for a single commodity flow. Recently, Combes (2011) studied this problem by minimizing the total landed cost using approximations and simulations. Dual sourcing also relates to the dual sourcing of commodities on the spot market and using forward contracts. Goel and Gutierrez (2011) provide an algorithm to study a dynamic dual basestock policy that depends on the spot price state. Our analysis and formulae may be applicable to similar settings with a constant cost differential between the two sources.

In this paper we study the order allocation to the global and local sources by introducing a class of order smoothing policies. Smoothing is a well known method to reduce variability. The benefit of order smoothing stems from the fact that the order pattern is less variable than the demand. Therefore, the total installed safety capacity is reduced compared to demand-replacing chase policies such as traditional base-stock policies. The introduction of order smoothing in a global dual sourcing context is not new. The TBS policy can actually be interpreted as an order smoothing policy: the presumption by Allon and Van Mieghem (2010) is that the low-cost source cannot rapidly change volumes because of frictions such as long leadtimes or an inflexible level production process which is essential to achieve this cost advantage. Indeed, under a TBS policy the global source needs no safety capacity. Moreover, an increase in the standing order reduces the variability of the responsive order stream ("peak-shaving behavior") and thus the required safety capacity of the responsive sources also reduces. Veeraraghavan and Scheller-Wolf (2008) specify a capacitated scenario in their dual-index policy with capacity limits at each source.

Smoothing is justified when production and holding costs are convex or when there is a cost of changing the level of production (Sobel 1969, 1971). Simon (1952) and Vassian (1955) did pioneering work on the development of smoothing rules using servomechanism (or control) theory and Laplace transform methods. Forrester (1961) and Magee (1958) suggest that production smoothing can be achieved by distributing the transient part of the required production over a number of successive periods. Bertrand (1986) extends this approach to a multi-product multi-phase production system.

Graves has contributed to the smoothing literature over the course of the last 25 years. Graves (1988) reviewed the literature on safety stocks for manufacturing systems and criticized its (lacking) consideration of the role of safety stocks in the presence of inflexibility in manufacturing systems. He characterized the need for additional safety stocks as a result of the smoothing or decoupling function within a manufacturing operation. Of particular interest to our work is the linear production control rule described in his paper which smoothes the aggregate production and permits an explicit examination of the tradeoff between safety stocks and production flexibility. A similar rule is used by Balakrishnan et al. (2004) who set the order quantity equal to a convex combination of the previously observed consumer demands. They make use of these order smoothing rules downstream in the chain to coordinate the entire supply chain. They also characterize the optimal smoothing parameter values and assess the potential cost savings that these order-smoothing strategies can yield compared to the uncoordinated case when individual firms separately minimize 
their costs. In our paper we use the same linear control rules to allocate orders to the global and local source, thereby smoothing production over both sources.

Recent empirical work on production smoothing by Cachon et al. (2007) found, based on industry-level US data, that order smoothing exists in the retail industry and in some manufacturing industries, but not in the wholesale industry. Chen and Lee (2012) show how the prevalence of capacity constraints in these industries (e.g., limited shelf/warehouse space and manufacturing capacity) drives order smoothing. Cantor and Katok (2012) use a series of laboratory experiments to demonstrate Cachon's (2007) findings: when the cost of varying orders is higher than the cost of holding inventory, production and order smoothing is indeed a rational and cost-minimizing behavior. Bray and Mendelson (2012) study firm-level US data and show that firms generally amplify last-minute shocks, yet smooth seasonal variations. Cui et al. (2014) present strong empirical evidence of order smoothing. There is also a large economics literature preceding the work in operations management, which empirically investigates production smoothing - we refer to Cachon et al. (2007) for an overview and discussion.

\section{Single Sourcing, Smoothing, and Capacity}

As a first stepping stone towards capacitated dual sourcing, this section sets up the full model and notation by reviewing single sourcing policies and discussing the impact of order smoothing when the source incurs capacity costs and has a general leadtime. Section 3.1. presents the full model and notation while the remainder of section 3 focuses on Single Sourcing. Dual sourcing policies are developed from Section 4 on.

\subsection{Sourcing Model}

Consider a periodic-review inventory system that can be replenished from two sources. (As a first stepping stone, we can source from one of these two sources; the next section will generalize to dual sourcing.) Time is discrete and the sequence of events at each time $t=0,1, \ldots, T$ is as follows: First the demand $D_{t}$ is observed and satisfied; unfilled demand is backlogged. Then, the net inventory $I_{t}$, which is the inventory on hand minus backorders, is observed and replenishment orders are placed. The analysis is simplified by letting $q_{t}^{i}$ denote the order quantity received in period $t$ from source $i$ with $i \in\{l, g\}$ for resp. the local and global source. The orders face a delay of resp. $L_{l}$ and $L_{g}$

periods, which means that the quantity $q_{t}^{i}$ that is received at time $t$ must be ordered in period $t-L_{i}$ (and thus depend only on quantities observed up to $t-L_{i}$ ). When $L_{i}=0$, the order is received in time to fill next period's demand; this is equivalent to saying that the replenishment is received by the end of the period in which its order is placed. Following Zipkin (2000, p. 404), we say that the risk period or total leadtime is $L_{i}+1$ periods (this risk period includes the one period review). The essence is that the sources have a leadtime difference of $L_{g}-L_{l}=L \geq 1$.

Demand is stationary and i.i.d. with $\mathbb{E}\left(D_{t}\right)=\mu, \operatorname{Var}\left(D_{t}\right)=\sigma^{2}$, and distribution $\Phi$. Let $\phi_{N}$ and $\Phi_{N}$ denote the standard normal density and distribution and $I_{N}(z)=\phi_{N}(z)-z\left(1-\Phi_{N}(z)\right)$ 
the unit normal loss function.

For the inventory evolution all that is needed is the demand process and the total quantity received $q_{t}=q_{t}^{l}+q_{t}^{g}$. Given this sequence of events where we first satisfy demand, then observe inventory and finally place and receive orders, we have the following dynamics of the net inventory for $t=1, \ldots, T$ :

$$
I_{t}=I_{t-1}+q_{t-1}-D_{t}
$$

The dynamics for the first period are $I_{0}=I_{-1}-D_{0}$. The initial inventory $I_{-1}$, which is a constant, can be decomposed into $I_{-1}=\sum_{i \in\{l, g\}}\left(L_{i}+1\right) \mathbb{E} q_{t}^{i}+I_{s}$, where $I_{s}$ denotes the safety-stock. In addition to the net inventory, there is an outstanding pipeline inventory $I_{t}^{p}=\sum_{i \in\{l, g\}} \sum_{k=0}^{L_{i}-1} q_{t+k}^{i}$.

The total landed cost per period incurred from origin (one of either source) to destination (finished goods warehouse) includes a cost per unit sourced, but also the capacity cost at source $i$, and the inventory (holding and shortage) costs. The total landed cost is most clear in a centralized system, where the global network with multiple supply points is wholly-owned and part of one organization. But also in a decentralized system, the supply capacity cost typically remains an important component of the total landed cost as the supplier would include a charge for it.

The sourcing cost equals $c^{i}$ per unit sourced from source $i$, with the faster source being more expensive $\left(c^{l}>c^{g}\right)$, reflects the standard variable cost component in the total cost for units coming from location $i$. This component includes direct material as well as any labor cost that can directly be attributed to the order size. We assume sourcing costs are incurred at receipt (although it doesn't make a difference in our undiscounted model).

The capacity cost at location $i$ reflects the standard fixed cost component in the total cost for units coming from location $i$ per period. This includes capital, labor, and other overhead cost rates that remain unchanged over the time horizon $[0, T]$. The installed capacity $K^{i}$ at source $i$ incurs a cost per period of $C\left(K^{i}\right)=k^{i} K^{i}$, with $k^{i}$ the constant, marginal cost rate to add one unit of capacity at source $i$. In the natural regime, local capacity is more expensive than global capacity (although the model works without those conditions). In practice, companies often have some volume flexibility to exceed the installed capacity which we model as follows: Orders $q_{t}^{i}$ can be produced up to the capacity $K^{i}$ that is installed at time 0 ; any excess order $\left(q^{i}-K^{i}\right)^{+}$ requires overtime capacity at extra cost $o^{i}$ per unit. Overtime reflects excess cost not covered in regular capacity costs nor standard direct labor. (Obviously, $k^{i}<o^{i}$, otherwise it would never be optimal to invest in capacity.) The ratio $o^{i} / k^{i}>1$ measures the rigidity of the capacity constraint; equivalently, $k^{i} / o^{i}<1$ can be interpreted as the degree of flexibility in quantity deviations beyond a source's installed capacity. The limit $o^{i} / k^{i} \rightarrow \infty$ represents the standard theoretical model of capacity as a hard constraint.

Finally, each period, inventory incurs a holding cost $h$ per unit on hand or a backlog cost $b$ per unit short. Given that we consider a wholly-owned global network, we also charge holding cost to the pipeline inventory as it represents capital tied up in the network (regardless whether the supplier or the buyer has the inventory on his accounts). The average cost over horizon $T$ becomes: 


$$
C_{I_{-1}}^{T}=\frac{1}{T} \sum_{i \in\{l, g\}} \sum_{t=0}^{T}\left[c^{i} q_{t}^{i}+k^{i} K^{i}+o^{i}\left(q_{t}^{i}-K^{i}\right)^{+}+h\left(I_{t}\right)^{+}+b\left(I_{t}\right)^{-}+h \sum_{k=0}^{L_{i}-1} q_{t+k}^{i}\right]
$$

We will focus on minimizing the average $\operatorname{cost} C=\lim _{T \rightarrow \infty} C_{I_{-1}}^{T}$. Ideally, one would like to characterize the initial inventory $I_{-1}$ and an admissible sourcing policy (which defines the replenishments $q_{t+L_{l}}^{l}$ and $q_{t+L_{g}}^{g}$ as a function of $I_{t-k}$ and $D_{t-k}$ for $\left.t, k=0,1, \ldots\right)$ that minimizes $C$. In the remainder of this section we review some principal single sourcing policies and their average $\operatorname{cost} C$ that will serve as a stepping stone towards dual sourcing policies.

\subsection{Standard single sourcing base-stock policy}

The standard single sourcing base-stock policy is optimal in minimizing inventory related costs only (Zipkin 2000). Although this policy is not optimal to minimize the total cost $C$ (which additionally includes sourcing and capacity costs), it is a well-known and useful benchmark against other sourcing policies. Single sourcing from either the local or the global source under a standard base-stock policy is a demand-replacement policy where $q_{t}^{i}=D_{t-L_{i}}$, and the associated inventory process is $I_{t}=I_{s}+\left(L_{i}+1\right) \mu-\sum_{i=t-L_{i}}^{t} D_{i}$. With normal demand, both the order and net inventory process are also normally distributed and the optimal capacity and safety stock levels then follow from a standard newsvendor solution:

$$
\begin{array}{lll}
K^{i *}=\mu+z_{K}^{i} \sigma & \text { where } & \Phi_{N}\left(z_{K}^{i}\right)=\frac{o^{i}-k^{i}}{o^{i}} \\
I_{s}^{*}=\sqrt{\left(L_{i}+1\right)} z_{I} \sigma & \text { where } & \Phi_{N}\left(z_{I}\right)=\frac{b}{b+h} .
\end{array}
$$

There is also pipeline inventory whose average cost follows from Little's law $\left(h L_{i} \mu\right)$. The total average cost of this single sourcing standard base-stock policy (which we denote by $s$ ) is

$$
C^{s}=c^{i} \mu+k^{i} \mu+\kappa^{i} \sigma+\sqrt{\left(L_{i}+1\right)} \kappa_{I} \sigma+h L_{i} \mu .
$$

with $\kappa^{i}$ and $\kappa_{I}$ resp. the financial capacity and inventory cost parameters:

$$
\begin{aligned}
\kappa^{i} & =k^{i} z_{K}^{i}+o^{i} I_{N}\left(z_{K}^{i}\right)=k^{i}\left[z_{K}^{i}+\frac{o^{i}}{k^{i}} I_{N}\left(z_{K}^{i}\right)\right], \\
\kappa_{I} & =h z_{I}+(h+b) I_{N}\left(z_{I}\right)=h\left[z_{I}+\frac{(h+b)}{h} I_{N}\left(z_{I}\right)\right] .
\end{aligned}
$$

The capacity cost parameter $\kappa^{i}$ increases linearly in the source's unit capacity cost $k^{i}$ and concavely in the capacity rigidity ratio $o^{i} / k^{i}$. The inventory cost parameter $\kappa_{I}$ increases in the holding cost $h$ and the critical fractile $z_{I}$ (or, equivalently, the ratio $b / h$ ).

Compared to single local sourcing, global single sourcing benefits from lower sourcing (and often capacity unit) costs, while the total order variability remains the same (the installed capacity will depend on the unit capacity cost and the rigidity of the source). In contrast, inventory costs 
increase due to the longer leadtime. To compare the two standard single sourcing policies, we consider the scaled cost

$$
\widehat{C}=\frac{C-\left(c^{l}+k^{l}+h L_{l}\right) \mu}{\kappa_{I} \sigma},
$$

and introduce the following dimensionless notation that also will be useful for dual sourcing:

$$
\theta_{l}=\frac{\kappa^{l}}{\kappa_{I}}, \theta_{g}=\frac{\kappa^{g}}{\kappa_{I}} \text { and } \theta_{c}=\frac{c^{l}-c^{g}+k^{l}-k^{g}-h L}{\kappa_{I}} \frac{\mu}{\sigma} .
$$

These three dimensionless parameters capture the "degrees of freedom" in the model and also the main trade-offs in devising a sourcing policy: $\theta_{l}$ and $\theta_{g}$ contain the ratio of resp. the local and global source's capacity cost and the rigidity of the source vs. the holding cost and the service level (see Eqs. (3-4) ). $\theta_{c}$ captures the unit cost advantage of the global source (in sourcing and unit capacity vis-a-vis the increased pipeline inventory cost), compared to the unit holding cost and the inventory service level (both through $\kappa_{I}$ ) and the volatility in demand (as measured by its coefficient of variation, $C V=\sigma / \mu$ ). To disentangle the impact of a change in $L$ or in cost differences between the sources, from here on we will have $\theta_{c}$ be the parameter capturing cost differences (meaning, if the leadtime difference increases, the absolute cost advantage must also increase to keep the cost difference $\theta_{c}$ the same). It directly follows that the scaled cost for local single sourcing $(l s)$ and global single sourcing ( $g s$ ) using the base-stock policy,

$$
\begin{aligned}
& \widehat{C^{l s}}=\theta_{l}+\sqrt{L_{l}+1}, \\
& \widehat{C^{g s}}=-\theta_{c}+\theta_{g}+\sqrt{L_{g}+1} .
\end{aligned}
$$

This directly leads to the following simple guideline to balance global and local sourcing when the standard base-stock policy is in use:

Proposition 1 With normal demand, and when using a standard base-stock replenishment policy, global single sourcing dominates local single sourcing if and only if $\theta_{c}+\theta_{l}-\theta_{g}>\sqrt{L_{g}+1}-\sqrt{L_{l}+1}$.

\subsection{Order Smoothing Policies with Single Sourcing}

The high cost of installed capacity has led to the development of ordering policies that dampen the variability in orders. One effective order policy uses exponential smoothing with smoothing level $\alpha \in[0,1]$. For an easy introduction to the order smoothing policy, first consider the case when the leadtime $L_{i}=0$. The order policy $q_{t}=\alpha q_{t-1}+(1-\alpha) D_{t}$, with $0 \leq \alpha \leq 1$ and $q_{-1}=\mu$ as an initial condition covers a set of policies that range between a chase and level strategy: If $\alpha=0$, then $q_{t}=D_{t}$ is a standard base-stock or demand-replacement (chase) policy, and if $\alpha=1$, then

$q_{t}=q_{t-1}=q_{-1}=\mu$ is a level strategy that orders the average demand each period. Any in-between smoothing level is a compromise between both and smoothes the orders.

Iterating the recursion shows that the total order quantity received in period $t$ is a linear 
combination of the observed demand process:

$$
q_{t}=\sum_{k=0}^{t}(1-\alpha) \alpha^{k} D_{t-k}+\alpha^{t+1} \mu
$$

This order policy finds its origin in linear control theory (Forrester 1961, Magee 1958). It is in essence a generalized base-stock policy, where the inventory deficit is not recovered in one period, but instead spread out over time, with $1 /(1-\alpha)$ the adjustment time to recover the deficit. The order smoothing policy also relates to the linear inflation rule that Zipkin (2000, p. 393) describes to deal with defects or yield losses. Graves (1988) used this control rule to smooth the aggregate production and it is also proposed by Balakrishnan et al. (2004) to reduce the variability in orders in a single source setting to reduce total supply chain costs (for $L_{i}=0$ ).

The smoothing policy (8) can be extended to general leadtimes $L_{i} \geq 0$. For $t \geq L_{i}$ :

$$
q_{t}=\sum_{k=L_{i}}^{t}(1-\alpha) \alpha^{k-L_{i}} D_{t-k}+\alpha^{t-L_{i}+1} \mu=\sum_{k=0}^{t-L_{i}}(1-\alpha) \alpha^{k} D_{t-L_{i}-k}+\alpha^{t-L_{i}+1} \mu .
$$

The linear control makes the policy analytically tractable. Taking expectations and variances of the orders yields:

$$
\mathbb{E} q_{t}=\mu, \quad \operatorname{Var}\left(q_{t}\right)=\left[1-\alpha^{2(t+1)}\right] \frac{1-\alpha}{1+\alpha} \sigma^{2} \leq \sigma^{2}
$$

The total order variance is convex decreasing in the smoothing level $\alpha$ and vanishes under the level strategy $\alpha=1$. As the order streams have smaller variability than the demand (which is referred to as smoothing), the optimal installed capacity reduces to

$$
K^{i *}=\mu+z_{K}^{i} \sigma \sqrt{\frac{1-\alpha}{1+\alpha}},
$$

and its corresponding capacity cost reduces to $C_{K}(\alpha)=k^{i} \mu+\kappa^{i} \sigma \sqrt{\frac{1-\alpha}{1+\alpha}}$. The reduction in capacity costs compared to the traditional base-stock policies represents the marginal benefit of order smoothing.

$$
M B(\alpha)=-C_{K}^{\prime}(\alpha)=\frac{1}{(1+\alpha) \sqrt{1-\alpha^{2}}} \kappa^{i} \sigma
$$

The linear order structure also yields analytic tractability of the inventory process: the net inventory process is a linear combination of the demand process, so with normal demand, the net inventory process $I_{t}$ is also normally distributed: (All proofs are relegated to the Technical Companion.)

Proposition 2 With normal demand, the net inventory process when single sourcing smoothing 
with leadtime $L_{i}$ is a linear combination of the demand process:

$$
\begin{aligned}
I_{t} & = \begin{cases}I_{-1}-\sum_{k=0}^{t-L_{i}} \alpha^{k} D_{t-L_{i}-k}-\sum_{k=0}^{L_{i}-1} D_{t-k} & \text { if } \alpha<1, \\
I_{-1}+t \mu-\sum_{i=0}^{t} D_{i}\end{cases} \\
\mathbb{E} I_{t} & =\begin{array}{ll}
I_{s} \alpha=1,
\end{array} \\
\lim _{t \rightarrow \infty} \operatorname{Var}\left(I_{t}\right) & = \begin{cases}\frac{1}{1-\alpha^{2}} \sigma^{2}+L_{i} \sigma^{2} \geq \sigma^{2} & \text { if } \alpha<1 \\
\infty & \text { if } \alpha=1 .\end{cases}
\end{aligned}
$$

The variance of the inventory process increases in the smoothing level $\alpha$ and grows without bound under level ordering with smoothing $(\alpha=1)$. (The source then supplies a constant quantity $\mu$ while demand remains random with same mean $\mu$. The resulting net inventory process behaves as a random walk with null drift and is unstable.) This increased inventory variance comes at a cost of requiring more safety inventory. The optimal safety stock level follows from a newsvendor solution: (This is not a standard newsvendor problem because the decision variable $I_{s}$ is the mean of the distribution but it can be reduced to a newsvendor model.)

Proposition 3 With normal demand and $\alpha<1$, the long-run optimal safety stock under order smoothing with general leadtime $L_{i}$ is

$$
I_{s}^{*}=z_{I} \sigma \sqrt{L_{i}+\frac{1}{1-\alpha^{2}}} .
$$

The associated inventory holding and backlogging cost rate, $C_{I}=\kappa_{I} \sigma \sqrt{L_{i}+\frac{1}{1-\alpha^{2}}}$ is convex increasing in $\alpha$, representing the marginal cost of smoothing.

$$
M C(\alpha)=C_{I}^{\prime}(\alpha)=\kappa_{I} \sigma \frac{\alpha}{\left(1-\alpha^{2}\right)^{\frac{3}{2}}\left(1+L_{i}-L_{i} \alpha^{2}\right)^{\frac{1}{2}}} .
$$

The total scaled cost of single sourcing smoothing (denoted by $s s$ ),

$$
\widehat{C^{s s}}(\alpha)=\theta_{i} \sqrt{\frac{1-\alpha}{1+\alpha}}+\sqrt{L_{i}+\frac{1}{1-\alpha^{2}}}
$$

is continuous in the interval $[0,1)$ with $\widehat{C^{s s}}(0)=\theta_{i}+\sqrt{1+L_{i}}$ and $\widehat{C^{s s}}(1)=+\infty$, and can be convex-concave-convex. There is, however, a unique minimum that satisfies the optimality condition $M B\left(\alpha^{*}\right)=M C\left(\alpha^{*}\right)$.

Proposition 4 With normal demand, and for any $0 \leq L_{i}$ and any $0<\theta_{i}$, there is a unique optimal smoothing level for order smoothing, satisfying the fixed point equation

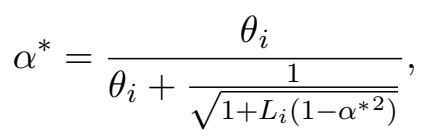



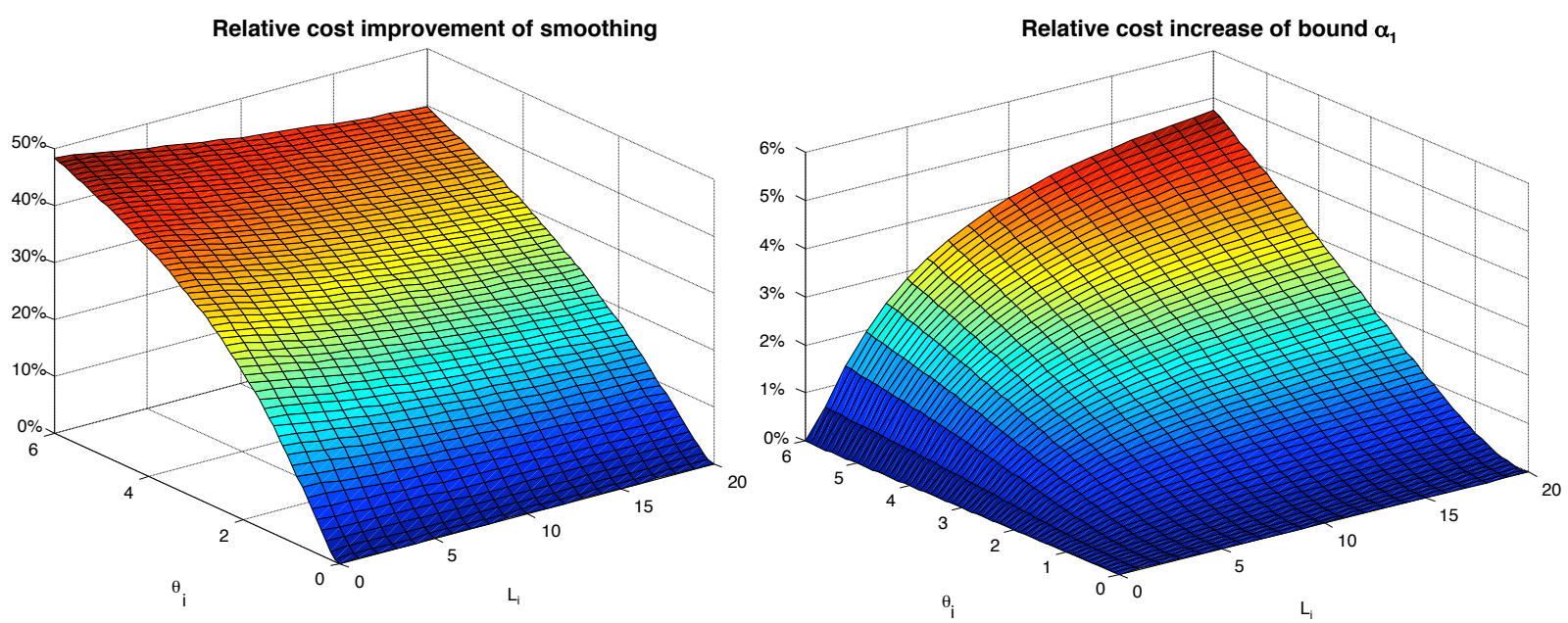

Figure 1: The value of order smoothing compared to the standard base-stock policy increases as the capacity $\operatorname{cost} \theta_{i}$ increases for any leadtime $L_{i}$ (left panel). The relative cost increase when bound $\alpha_{1}$ is used instead of the optimal $\alpha^{*}$ is small (right panel).

which is bounded by

$$
\alpha_{0}=\frac{\theta_{i}}{\theta_{i}+1} \leq \alpha^{*} \leq \alpha_{1}=\frac{\theta_{i}}{\theta_{i}+\left(1+L_{i}\right)^{-\frac{1}{2}}}
$$

Order smoothing sourcing outperforms the standard single sourcing base-stock policy for any $L_{i}$ if $\theta_{i}>0$. The value of smoothing increases in $\theta_{i}$ if leadtime $L_{i}=0$.

If $L_{i}=0$, Eq. 11 reduces to $\alpha^{*}=\frac{\theta_{i}}{1+\theta_{i}}$ and its scaled cost $\widehat{C^{s s}}\left(\alpha^{*}\right)=\sqrt{1+2 \theta_{i}}$. The relative cost benefit of smoothing compared to the standard base-stock can be quantified as

$$
0 \leq \frac{\widehat{C^{s}}-\widehat{C^{s s}}}{\widehat{C^{s}}}=1-\frac{\sqrt{1+2 \theta_{i}}}{1+\theta_{i}} \leq 1-\frac{1}{\sqrt{1+\theta_{i}}}
$$

which increases in $\theta_{i}$ towards a maximum of $100 \%$. Figure 1 s left panel shows that the relative cost improvement of order smoothing compared to using the standard base-stock policy holds for leadtimes $L_{i} \geq 0$. When capacity is costly, the value of order smoothing can be substantial 2

Based on (12), we can derive the following bounds on the optimal cost (Figure 1b shows the

\footnotetext{
${ }^{2}$ Whereas Figure 1 compares order smoothing with the standard base-stock using the same source, one also can similarly compare order smoothing from one source with the standard base-stock policy from another source using Eqs. (7) and (13). As such, it may for instance turn out to be beneficial for a firm that faces capacity supply costs to smooth orders from the local source, rather than source globally at low cost.
} 
accuracy of $\widehat{C^{s s}}\left(\alpha_{1}\right)$ compared to the true optimal costs $\left.\widehat{C^{s s}}\left(\alpha^{*}\right)\right)$ :

$$
\begin{aligned}
& \widehat{C^{s s}}\left(\alpha_{0}\right)=\theta_{i} \sqrt{\frac{1}{1+2 \theta_{i}}}+\sqrt{L_{i}+\frac{\left(1+\theta_{i}\right)^{2}}{1+2 \theta_{i}}}, \\
& \widehat{C^{s s}}\left(\alpha_{1}\right)=\theta_{i} \sqrt{\frac{1}{1+2 \theta_{i} \sqrt{1+L_{i}}}}+\sqrt{L_{i}+\frac{\left(1+\theta_{i} \sqrt{1+L_{i}}\right)^{2}}{1+2 \theta_{i} \sqrt{1+L_{i}}}} .
\end{aligned}
$$

The right panel of Figure 1 shows that the cost penalty of using bound $\alpha_{1}$ is modest.

\subsection{Optimal Capacitated Single Sourcing}

Although the order smoothing policy does not guarantee optimality in minimizing the total landed $\operatorname{cost} C$, its linear structure makes it attractive due to its analytic tractability. This allows us to derive closed form solutions, and comes with the benefit of gaining insight into the cost drivers of the sourcing policy. For $L_{i}=0$, we can numerically show that order smoothing closely tracks the optimal policy. The presence of capacity costs as modeled above, is actually equivalent to piece-wise linear convex order costs. The associated optimal policy for $L_{i}=0$ is then characterized by a dual base-stock policy. (For $L_{i}>0$ the optimal policy optimal policy uses the full history of orders and is therefore more complex.) When the inventory position exceeds the higher base-stock level, no order is placed. When the inventory position is below the higher base-stock, we first use up the regular capacity $K$. If this raises the inventory position to above the lower base-stock, we do not use overtime; otherwise, we use overtime to raise the inventory position to the lower base-stock level. In other words, there is a region of "inaction" where we order maximal $K$ but less than the demand. (The marginal overtime cost exceeds the marginal benefit of raising inventory in terms of reducing backlogging relative to holding. In other words, it is better to wait and replenish in

the future at regular cost versus now at overtime cost.) This is not a demand replacing policy and there are no simple solutions for the optimal base-stock levels and capacity level $K$.

Given that SCC cannot be optimized analytically, we numerically optimized its simulated cost. Figure 2 shows the total scaled cost of local single sourcing when $L_{i}=0$ using the standard base-stock policy, using order smoothing and using the optimal dual base-stock described above. Although order smoothing does not guarantee optimality, it proves its value in the presence of capacity costs and closely tracks the optimal policy.

\section{Dual Sourcing and Order Smoothing}

\subsection{Dual sourcing model}

Consider the dual sourcing setting where units can be ordered from a local source and/or from a global source. As before, let $i \in\{l, g\}$ refer to the local or the global source; in particular, $L_{i}$ 


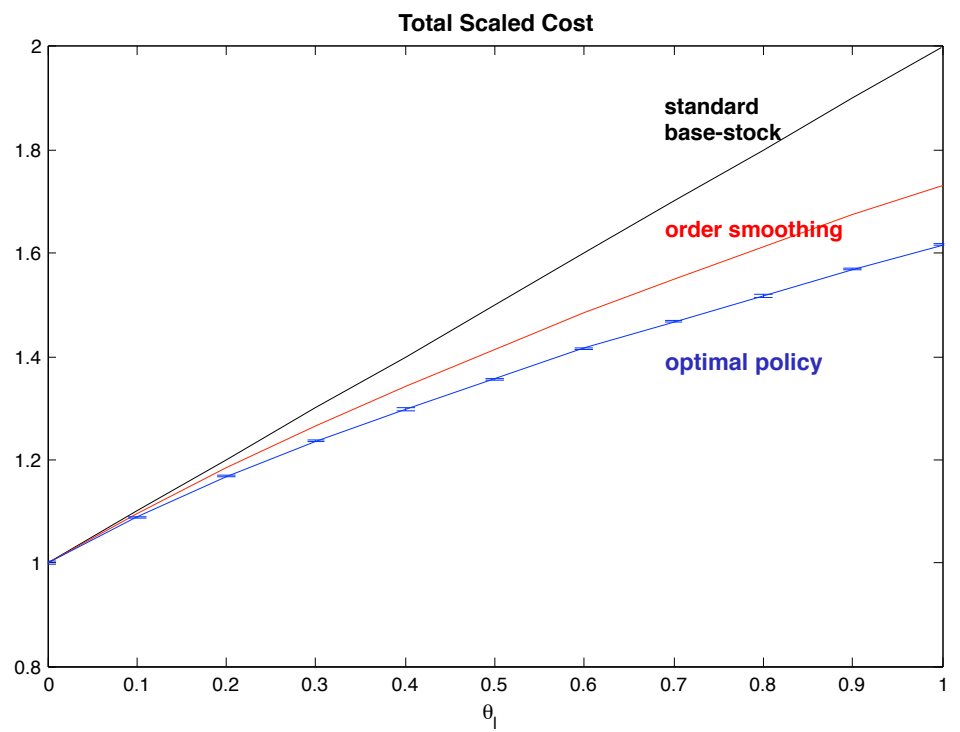

Figure 2: Single sourcing using the standard base-stock policy, order smoothing and the optimal capacitated dual base-stock policy (with $95 \%$ confidence intervals) when $L_{i}=0$.

denotes the leadtime of source $i$, where $0 \leq L_{l}<L_{g}$. With two sources, we decompose the total order quantity received in period $t$ as $q_{t}=q_{t}^{l}+q_{t}^{g}$, where $q_{t}^{i}$ denotes the order quantity received in period $t$ from source $i$. With leadtimes $L_{l}<L_{g}$, the received quantity $q_{t}^{i}$ is based on information older than $L_{i}$ periods. Correspondingly, we define a dual sourcing smoothing (DSS) policy by splitting up the total smoothed order stream $q_{t}$ as follows:

$$
\begin{aligned}
q_{t}^{g} & =\sum_{k=L_{g}}^{t}(1-\alpha) \alpha^{k-L_{l}} D_{t-k}=\sum_{k=L}^{t-L_{l}}(1-\alpha) \alpha^{k} D_{t-L_{l}-k} \\
q_{t}^{l}=\sum_{k=L_{l}}^{L_{g}-1}(1-\alpha) \alpha^{k-L_{l}} D_{t-k} & =\sum_{k=0}^{L-1}(1-\alpha) \alpha^{k} D_{t-L_{l}-k}
\end{aligned}
$$

where $L=L_{g}-L_{l}>0$ denotes the leadtime difference between both sources. Observe that for any leadtime difference $L>1$ between the global and local source, local orders placed within $L-1$ units of time after a global order will be received prior to the global order's receipt. Our model thus includes order crossing. Given that our orders are a linear combination of the demands only, they do not depend on any state variables or interaction between local and global inventory position. Therefore, order crossing does not pose any problems or complications to our analysis.

For $t \rightarrow \infty$, the variability in orders is independent of $L_{l}$ (and the same as when $L_{l}=0$ ):

$$
\begin{array}{ll}
\mathbb{E} q_{t}^{g}=\alpha^{L} \mu, & \operatorname{Var}\left(q_{t}^{g}\right)=\frac{1-\alpha}{1+\alpha} \alpha^{2 L} \sigma^{2}, \\
\mathbb{E} q_{t}^{l}=\left(1-\alpha^{L}\right) \mu, & \operatorname{Var}\left(q_{t}^{l}\right)=\frac{1-\alpha}{1+\alpha}\left(1-\alpha^{2 L}\right) \sigma^{2} .
\end{array}
$$

Notice that $(i)$ the strategic allocation $a$ (the fraction of average total orders allocated to the global source) is $\alpha^{L}$, which is different and smaller than the smoothing level $\alpha$; (ii) The variance of each 
order stream is less than the demand variance (consistent with "order smoothing"). The total order variance is convex and decreasing in the smoothing level $\alpha$ and vanishes under the level strategy $\alpha=1$ (i.e., single sourcing with constant order $q_{t}=q_{t}^{g}=\mu$ from the global source). The pipeline inventory holding cost is $h L_{g} \alpha^{L} \mu+h L_{l}\left(1-\alpha^{L}\right) \mu=h L_{l} \mu+h L \alpha^{L} \mu$. The inventory dynamics under this dual sourcing smoothing policy equal those under single sourcing smoothing with leadtime $L_{l}$ (because total receipts $q_{t}=q_{t}^{l}+q_{t}^{g}$ are equal). Thus, the inventory is independent of the leadtime difference $L$ but it does depend on the local leadtime $L_{l}$ :

$$
\operatorname{Var}\left(I_{t}\right)=\frac{1}{1-\alpha^{2}} \sigma^{2}+L_{l} \sigma^{2}
$$

The total absolute and scaled (as defined by equation (5) costs thus become:

$$
\begin{aligned}
C= & \left(c^{g}+k^{g}\right) \alpha^{L} \mu+\left(c^{l}+k^{l}\right)\left(1-\alpha^{L}\right) \mu+\kappa^{g} \sigma \alpha^{L} \sqrt{\frac{1-\alpha}{1+\alpha}}+\kappa^{l} \sigma \sqrt{\frac{1-\alpha}{1+\alpha}\left(1-\alpha^{2 L}\right)} \\
& +\kappa_{I} \sigma \sqrt{L_{l}+\frac{1}{1-\alpha^{2}}}+h L_{l} \mu+h L \alpha^{L} \mu, \\
\widehat{C}= & -\theta_{c} \alpha^{L}+\theta_{g} \alpha^{L} \sqrt{\frac{1-\alpha}{1+\alpha}}+\theta_{l} \sqrt{\frac{1-\alpha}{1+\alpha}\left(1-\alpha^{2 L}\right)}+\sqrt{L_{l}+\frac{1}{1-\alpha^{2}}} .
\end{aligned}
$$

The trade-offs are clearly shown in Fig. 3. A higher smoothing level $\alpha$ implies a larger reliance on the global source and reduces sourcing and local capacity costs but increases inventory costs. (Global capacity costs initially increase reflecting a higher needed safety capacity as the global allocation increases; yet they decrease to zero as $\alpha \rightarrow 1$, which corresponds to a standing constant order $q_{t}^{g}=\mu$ and no safety capacity is needed.) This directly raises the question whether there is an optimal trade-off and, if so, how to characterize it. To that end, let $\widehat{C}^{*}=\widehat{C}\left(\alpha^{*}\right) \leq \widehat{C}(0)=$ $\theta_{l}+\sqrt{L_{l}+1}$ denote the minimal cost (which exists because $\widehat{C}(\alpha)$ is continuous in the interval $[0,1$ ) with $\widehat{C}(1)=\infty)$ and $\alpha^{*} \in[0,1)$ an optimal smoothing level. Similarly, let $a^{*}=\left(\alpha^{*}\right)^{L}$ denote the corresponding strategic allocation.

\subsection{Impact of Sourcing Cost Difference $\theta_{c}$ and Leadtimes}

To highlight the impact of the cost and leadtime differences between both sources, we first consider the uncapacitated system where $\theta_{l}=\theta_{g}=0$. (Recall that, to disentangle the impact of both, $\theta_{c}$ only captures the cost difference.) As Figure 4 illustrates, the total cost and thus optimal trade-off depend jointly on the smoothing level $\alpha$ and leadtime difference $L$ in non-obvious ways: First, the cost increases as the leadtime difference $L$ increases and the boundary solution $\alpha^{*}=0$ is optimal above a certain threshold value of $L$ (which is $L=4$ in Figure 4 ). Second, while the cost is convex for $L=1$, it is concave-convex for $L=2$, and convex-concave-convex for $L>2$ and can have a local maximum and minimum (as illustrated for $L=3$ ). Third, the optimal smoothing level is not monotone in $L$, but the optimal strategic allocation is. We will generalize these observations in this section. 


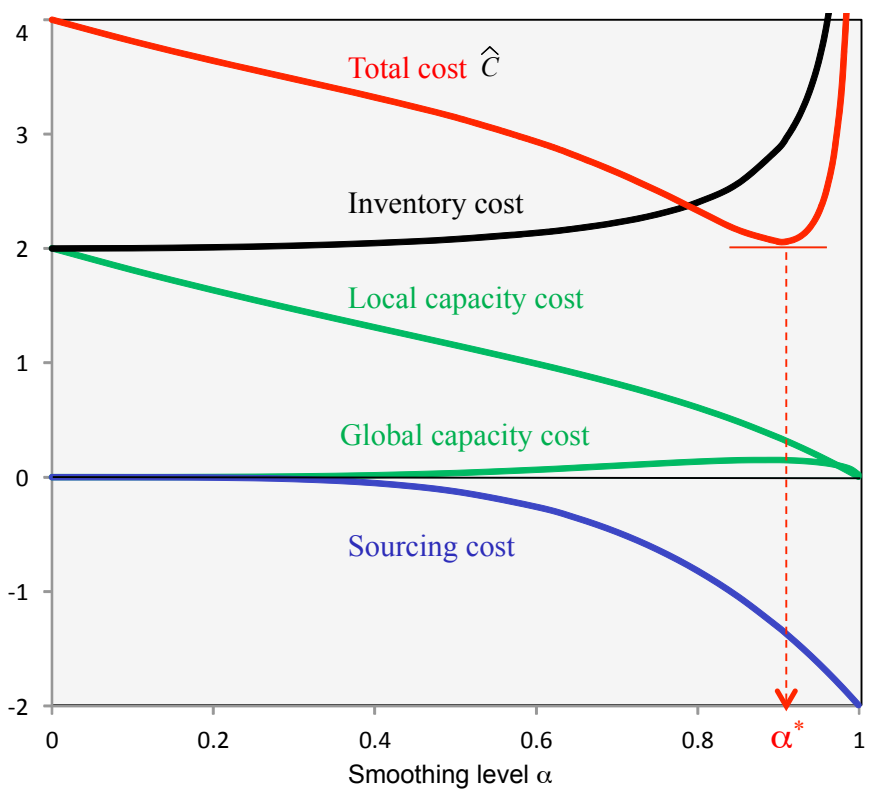

Figure 3: More smoothing implies a larger reliance on the global source which decreases sourcing and local capacity costs but increases inventory costs. There is a unique optimal smoothing level $\alpha^{*}$ or offshoring allocation $\alpha^{* L}$. (Parameters: $\theta_{c}=2, \theta_{l}=2, \theta_{g}=1, L_{l}=3, L_{g}=7$ ).

Optimal dual sourcing smoothing requires that $\alpha^{*} \in(0,1)$ where $\alpha^{*}$ satisfies the first order condition (FOC):

$$
M B\left(\alpha^{*}\right)=M C\left(\alpha^{*}\right) \Leftrightarrow L \theta_{c}\left(\alpha^{*}\right)^{L-1}=\frac{\alpha^{*}}{\left(1-\alpha^{* 2}\right)^{\frac{3}{2}}\left(1+L_{l}\left(1-\alpha^{* 2}\right)\right)^{\frac{1}{2}}} .
$$

Manipulating the FOC shows the impact of three essential parameters:

Proposition 5 Under our model assumptions, as the local leadtime $L_{l}$ or the leadtime difference $L$ increases, the optimal cost $\widehat{C^{*}}$ increases. The optimal offshoring allocation $a^{*}$ increases as $L_{l}$ increases, but decreases as $L$ increases. As the standardized sourcing cost advantage $\theta_{c}$ increases, the optimal cost $\widehat{C^{*}}$ decreases at rate $-1<\frac{d}{d \theta_{c}} \widehat{C}\left(\alpha^{*}\right)=-\alpha^{* L}<0$ and the optimal smoothing level $\alpha^{*}$, and thus offshoring allocation $a^{*}$, increases.

By investigating how model parameters impact $\theta_{c}$ and $L$, the proposition confirms the intuitive impact of these parameters on the optimal offshoring allocation $\alpha^{* L}$ and cost:

1. As expected, if the global sourcing unit cost advantage $c^{l}-c^{g}$ increases, then $\theta_{c}$ and thus $\alpha^{* L}$ increase while cost decreases.

2. If the demand volatility, measured by its coefficient of variation $C V=\sigma / \mu$, increases, then $\theta_{c}$ and thus $\alpha^{* L}$ decrease while the cost increases proportionally. 

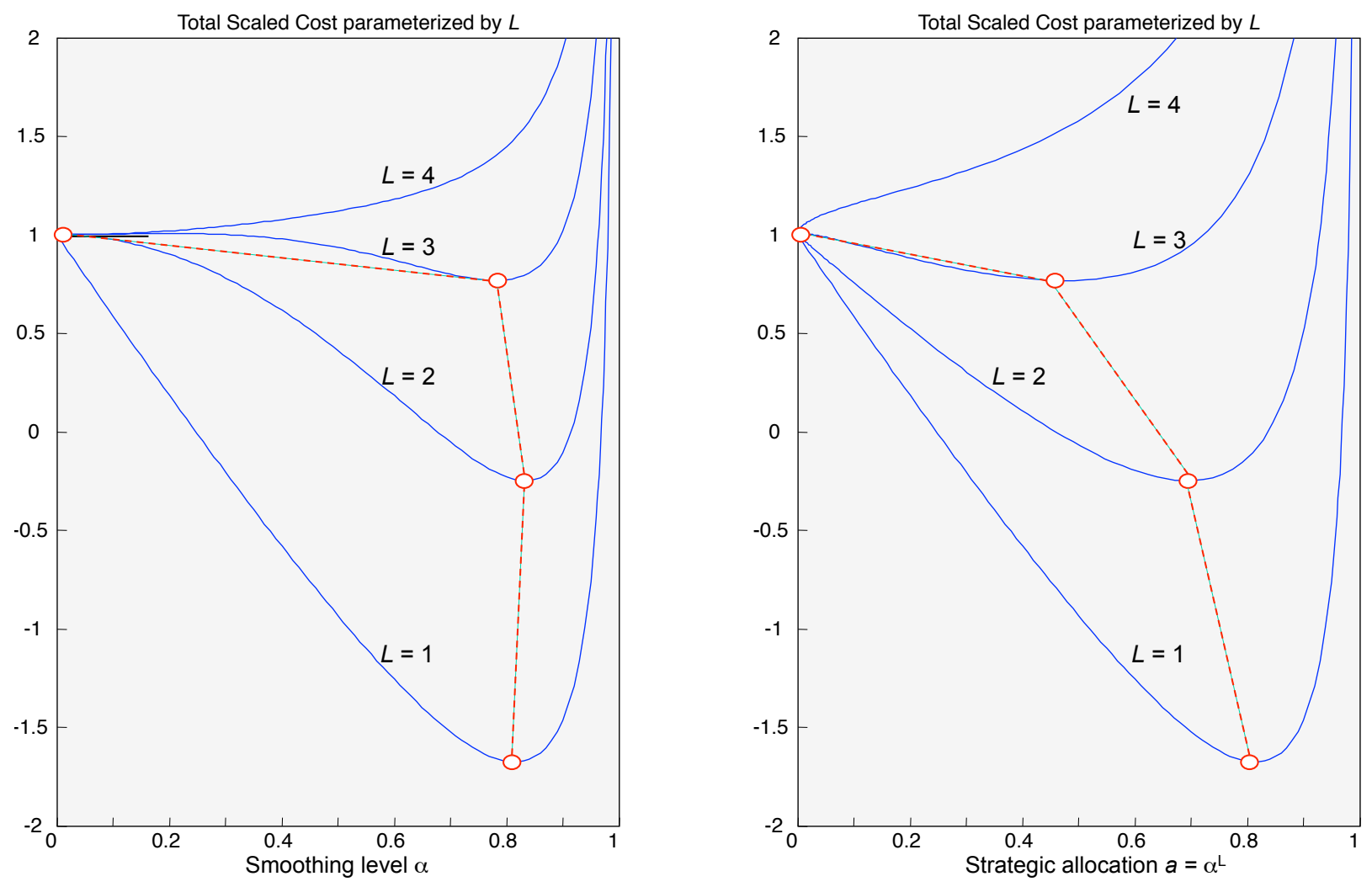

Figure 4: Total cost and corresponding optimal smoothing level $\alpha^{*}$ as a function of $L$ (left panel). The optimal strategic allocation $\alpha^{L}$ decreases as $L$ increases (right panel). (Both shown for $L_{l}=0$.)

3. If $h$ increases, then $\kappa_{I}$ increases ${ }^{3}$ so that $\theta_{c}$ and thus $\alpha^{* L}$ decrease and cost increases.

These comparative statics give guidance on how to tailor and adapt the sourcing strategy to the financial, customer service, and demand characteristics. The tractability of our model allows additional insight and quantification: Substituting $x=1-\alpha^{2}$ in the first order condition (16) yields

$$
f(x)=x^{3}\left(1+L_{l} x\right)(1-x)^{L-2}=\left(L \theta_{c}\right)^{-2} .
$$

Before we specify the optimal smoothing level and offshoring allocation in exact analytic terms, notice that $x^{*}=1-\alpha^{* 2}$ is easily found graphically as the intersection of the horizontal line at $\left(\theta_{c} L\right)^{-2}$ with the upward part of $f$ in Figure 5. As $f$ has a maximum for $L \geq 3$, the existence of this intersection (and thus an interior solution to the FOC) requires that $\left(\theta_{c} L\right)^{-2}$ does not exceed the maximum of $f$, which gives a lower bound $\underline{\theta}_{L, L_{l}}$ on $\theta_{c}$. Also, the maximizer of $f$ provides an upper bound on $x^{*}$, and thus lower bound $\underline{\alpha}_{L, L_{l}}$ on $\alpha^{*}$. (Proposition 17 on Technical Companion Page 15 provides expressions for these bounds.) Whereas $\theta_{c} \geq \underline{\theta}_{L, L_{l}}$ guarantees that the FOC have an interior solution $\alpha^{*}>0$, for dual sourcing smoothing to be optimal, this local cost minimum

\footnotetext{
$3 \frac{d \kappa_{I}}{d h}=\frac{d}{d h}\left[h z_{I}+(h+b) I_{N}\left(z_{I}\right)\right]=\phi_{N}\left(z_{I}\right)+\Phi_{N}\left(z_{I}\right) z_{I}>0$ for all $z_{I}$ as optimal functions of $h$.
} 


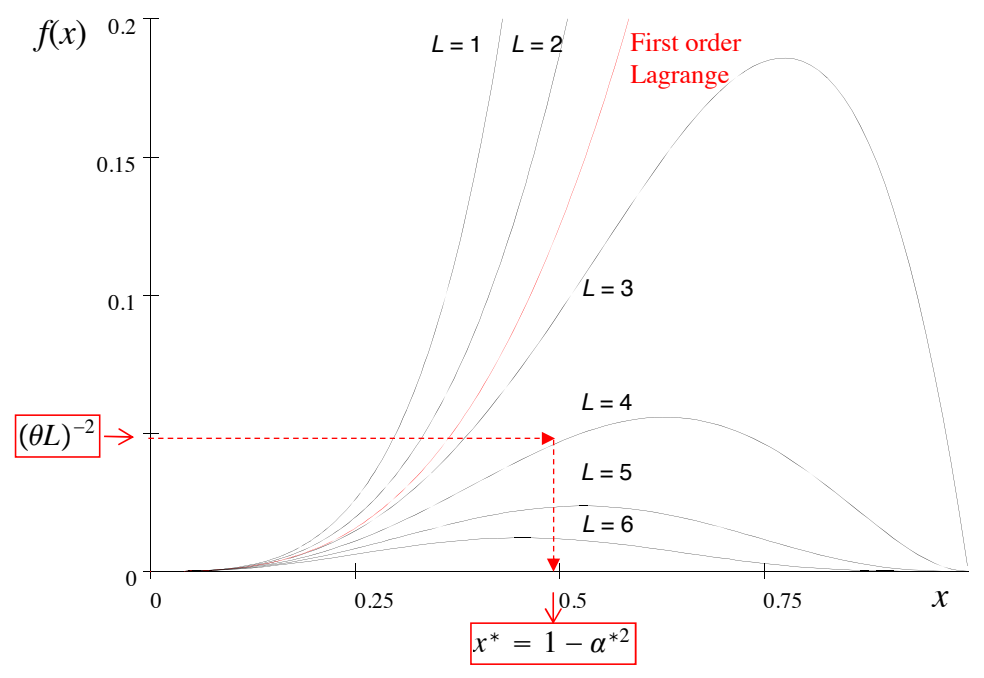

Figure 5: The optimal $x^{*}=1-\alpha^{* 2}$ is easily found graphically as a $f^{-1}\left(\left(\theta_{c} L\right)^{-2}\right)$, shown here for $L_{l}=1$.

must have a cost below the single sourcing cost $\widehat{C}(0)=\sqrt{L_{l}+1}$. This requires a more stringent condition $\theta_{c} \geq \underline{\theta}_{L, L_{l}}^{*}=\inf \left\{\theta_{c}: \widehat{C}\left(\alpha^{*} ; \theta_{c}, L\right)<\sqrt{L_{l}+1}\right\} \geq \underline{\theta}_{L}$.

For $L_{l}=0$ and $L=2$, Eq. (17) has a simple, unique solution if $\left(2 \theta_{c}\right)^{-2} \leq 1: x^{*}=\left(\theta_{c} L\right)^{-\frac{2}{3}}$ so that

$$
\alpha^{*}=\sqrt{1-\left(2 \theta_{c}\right)^{-\frac{2}{3}}} \text { and } \widehat{C}\left(\alpha^{*}\right)=\frac{3}{2}\left(2 \theta_{c}\right)^{\frac{1}{3}}-\theta_{c} .
$$

Other special cases include: if $L_{l}=0$ and $L=1$, this is a cubic equation with a unique root in $[0,1)$ for any $\theta_{c} \geq 0$ that can be solved exactly using the Cardano-Tartaglia formula. For $L \geq 3$, the cost function is convex-concave-convex and the FOC has two solutions in $[0,1]$, representing two local extrema $x^{*}$. The local minimum corresponds to the smallest root $x^{*}$ of a polynomial of degree $L+2$ if $L_{l}>0$ and of degree $L+1$ otherwise. If the degree is 4 , the root of the quartic can be solved using Ferarri's formula. (The Cardano and Ferrari formulas are relegated to the Technical Report.) If the FOC is a polynomial of degree greater than 4, Galois (1846) showed that there exists no general "simple" formula (i.e., using only a finite number of the usual algebraic operations and radicals) to specify its root $x^{*}$. Below we apply the inversion theorem of Lagrange (1770) which provides a Taylor series, expanded around $x_{0}$, for the inverse of an analytic function. We set $x_{0}=0$ and use this powerful technique to specify the root $f^{-1}\left(\left(\theta_{c} L\right)^{-2}\right)$ as an infinite series. (The Appendix shows the derivation.) We further show that its first order term is a bound that is asymptotically correct as $L \theta_{c} \rightarrow \infty$.

Proposition 6 Under normal demand and without capacity costs $\left(\theta_{g}=\theta_{l}=0\right)$, the optimal 
smoothing level $\alpha^{*}=0$ if $\theta_{c} \leq \underline{\theta}_{L, L_{l}}^{*}$, otherwis£ $\varliminf^{4}$

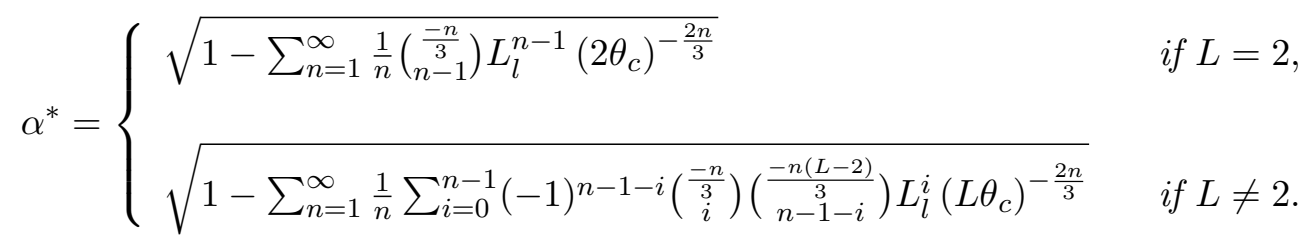

The Lagrange series $(19)$ shows that the leadtime difference $L$ has a first order impact, whereas $L_{l}$ only has a second order impact: $\alpha^{*} \simeq \sqrt{1-\left(L \theta_{c}\right)^{-\frac{2}{3}}+\frac{\left(L-L_{l}-2\right)}{3}\left(L \theta_{c}\right)^{-\frac{4}{3}}}$. In addition, its first order term provides a simple approximation for $\alpha^{*}$, which is also a bound for $L_{l}=0$ or 1 as illustrated in Fig. 5. (For $L_{l} \geq 2$, the first-order approximation of $f^{-1}$ no longer cleanly partitions the exact $f^{-1}$ for different values of $L$.)

Proposition 7 With normal demand and if $L \theta_{c}>1$, then the optimal smoothing level $\alpha^{*}$ has a square root approximation $\alpha_{0}$,

$$
\alpha_{0}=\sqrt{1-\left(L \theta_{c}\right)^{-\frac{2}{3}}}
$$

that is asymptotically correct: $\alpha_{0} \rightarrow \alpha^{*}$ as $L \theta_{c} \rightarrow \infty$. If $L_{l}=0$ or 1 , the approximation is a lower bound $\left(\alpha_{0} \leq \alpha^{*}\right)$ if $L \leq 2$ and is an upper bound $\left(\alpha_{0} \geq \alpha^{*}\right)$ if $L \geq 3$. It is exact if $L=2$ and $L_{l}=0$. Similarly, the optimal cost $\widehat{C}\left(\alpha^{*}\right)$ has an upper bound $\widehat{C}\left(\alpha_{0}\right)$ that is exact for $L=2$ and $L_{l}=0$ and asymptotically correct as $L \theta_{c} \rightarrow \infty$ wher $5^{5}$

$$
\widehat{C}\left(\alpha_{0}\right)=-\theta_{c}\left(1-\left(L \theta_{c}\right)^{-2 / 3}\right)^{L / 2}+\left(L_{l}+\left(L \theta_{c}\right)^{2 / 3}\right)^{\frac{1}{2}}=\frac{3}{2}\left(L \theta_{c}\right)^{\frac{1}{3}}-\theta_{c}-O\left(\left(L-2-4 L_{l}\right)\left(L \theta_{c}\right)^{-\frac{1}{3}}\right) .
$$

Fig. 6 compares the square root allocation $a_{0}=\alpha_{0}^{L}$ with the optimal allocation $a^{*}=\alpha^{* L}$ for $L=1$ (left panel) and $L=3$ (right panel), both for $L_{l}=0$. In addition to providing a fine approximation of the allocation, the square root formula's cost penalty $\widehat{C}\left(\alpha_{0}\right)-\widehat{C}\left(\alpha^{*}\right)$ is very low: for $L=3$, its maximal cost penalty is 0.027 (at $\theta_{c}=\underline{\theta}_{3}^{*}=1.15$ when $\widehat{C}\left(\alpha^{*}\right)=1$ ) and diminishes quickly as $\theta_{c}$ increases (it is .011 at $\theta_{c}=2$, and .003 at $\theta_{c}=5$ ). For $L=1$, the cost penalty starts out higher at 0.35 (at $\theta_{c}=1$ when $\widehat{C}\left(\alpha_{0}\right)=1$ ) but again diminishes quickly to 0.04 at $\theta_{c}=2$ and to 0.01 at $\theta_{c}=5$. For $L=1$, an expansion of the FOC for $\theta_{c}$ near 0 gives a better lower bound $\alpha_{1}=\frac{\theta_{c}}{\sqrt{1+3 \theta_{c}^{2}}}$ (shown in green).

\footnotetext{
${ }^{4}$ The generalized binomial coefficient is defined for $x \in \mathbb{R}:\left(\begin{array}{l}x \\ 0\end{array}\right)=1$ and $\left(\begin{array}{l}x \\ k\end{array}\right)=\frac{x(x-1) \cdots(x-k+1)}{k !}$ for $k=1,2, \cdots$.

${ }^{5}$ The Landau notations specify functions $o(f)$ that are of smaller order than $f$, and $O(g)$ which is of similar order as $g$. Formally: $\lim _{x \rightarrow \infty} O(f)(x) / f(x)=0$ while $\lim _{x \rightarrow \infty} O(g)(x) / g(x)$ is a finite constant.
} 

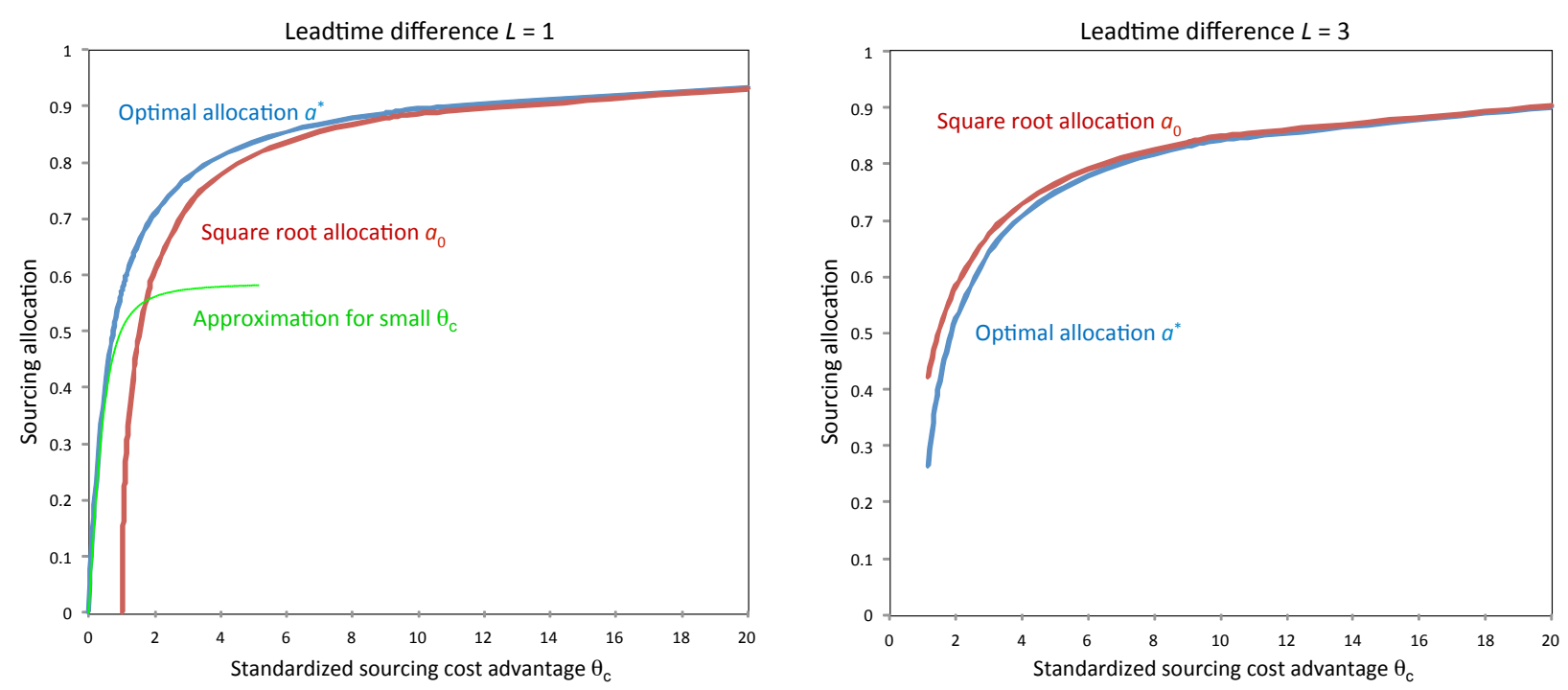

Figure 6: The optimal allocation $a^{*}=\alpha^{* L}$ compared to the square root allocation $a_{0}=\alpha_{0}^{L}$ for $L=1$ (left panel) and $L=3$ (right panel); both for $L_{l}=0$.

The square root approximation and bound also highlights the first-order impact of the standardized sourcing cost advantage $\theta_{c}$ and the leadtime difference $L$ on the global allocation:

Global (offshoring) allocation $a_{0}=\alpha_{0}^{L}=\sqrt{\left(1-\left(L \theta_{c}\right)^{-\frac{2}{3}}\right)^{L}}=\left(1-\left(\frac{c^{l}-c^{g}-h L}{\kappa_{I} C V} L\right)^{-\frac{2}{3}}\right)^{\frac{L}{2}}$

Figure 7 depicts this first order impact of the leadtime difference and variability (as measured by the $C V$ ) on offshoring.

\subsection{Impact of Capacity Costs $\theta_{l}$ and $\theta_{g}$}

With capacitated sources, any optimal dual sourcing smoothing level $\alpha^{*} \in(0,1)$ satisfies $\widehat{M B}\left(\alpha^{*}\right)=$ $\widehat{M C}\left(\alpha^{*}\right)$ where the marginal inventory cost remains as before, but the marginal benefit is augmented with the marginal capacity benefits:

$$
\widehat{M B}(\alpha)=\theta_{c} L \alpha^{L-1}-\theta_{g} \alpha^{L-1} \frac{L(1-\alpha)(1+\alpha)-\alpha}{(1-\alpha)^{\frac{1}{2}}(1+\alpha)^{\frac{3}{2}}}+\theta_{l} \frac{L \alpha^{2 L-1}(1-\alpha)(1+\alpha)+\left(1-\alpha^{2 L}\right)}{\left(1-\alpha^{2 L}\right)^{\frac{1}{2}}(1-\alpha)^{\frac{1}{2}}(1+\alpha)^{\frac{3}{2}}}
$$

Notice that the marginal global capacity benefit (term in $\theta_{g}$ ) is the only term that can be negative (for small smoothing levels $\alpha<\frac{\sqrt{1+4 L}-1}{2 L}$ ). We have the following comparative statics:

Proposition 8 Under our model assumptions, as the leadtime difference $L$ increases, the optimal cost $\widehat{C^{*}}$ increases if $\theta_{c} \geq \theta_{l}+\theta_{g}$. The optimal smoothing level and offshoring allocation increase when $\theta_{c}$ or $\theta_{l}$ increases, and decrease when $\theta_{g}$ increases if $\alpha^{*} \leq \frac{\sqrt{1+4 L}-1}{2 L}$. However, if $\alpha^{*}>\frac{\sqrt{1+4 L}-1}{2 L}$, the optimal smoothing level and offshoring allocation increase when $\theta_{g}$ increases. The corresponding 


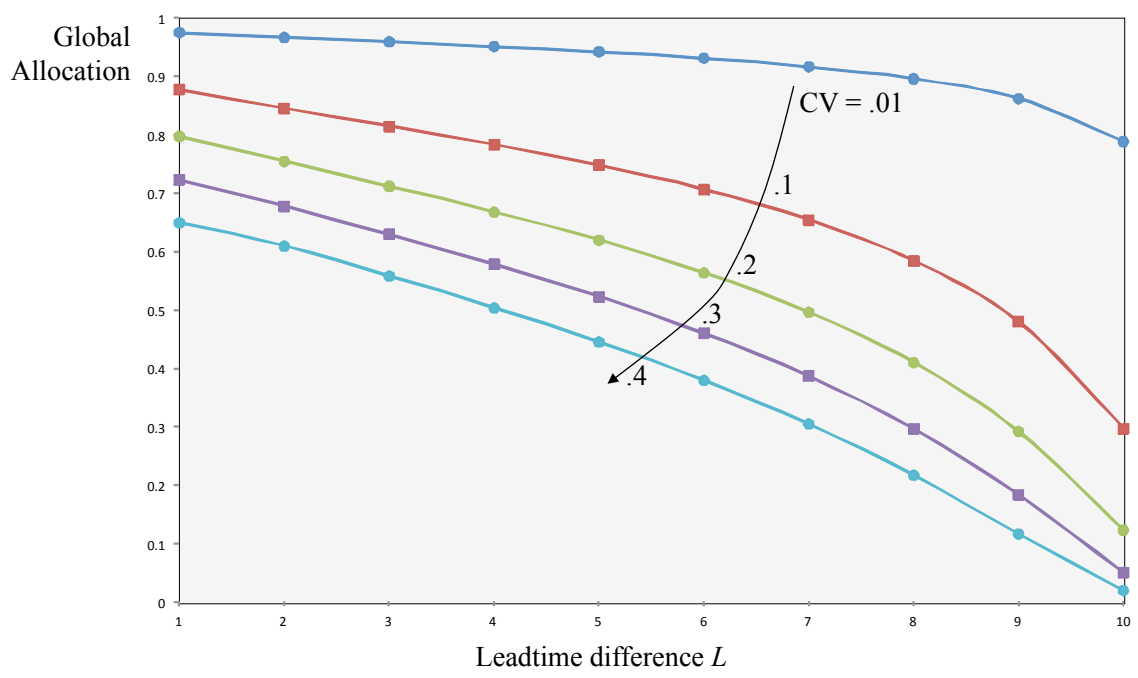

Figure 7: The impact of leadtime difference $L$ and coefficient of variation $C V$ on the global allocation $a_{0}=\alpha_{0}^{L}$. (Parameters: $c^{l}-c^{g}=1, h=0.09, \kappa_{I}=1$.)

rate of change in the optimal cost $\widehat{C^{*}}$ is bounded by the optimal allocation $\alpha^{* L}<1$ or by 1 :

$$
\begin{aligned}
& \frac{d \alpha^{*}}{d \theta_{c}} \geq 0 \\
& \frac{d \alpha^{*}}{d \theta_{l}} \geq 0 \\
& \frac{d \alpha^{*}}{d \theta_{g}}=\left\{\begin{array}{c}
\leq 0 \text { if } \alpha^{*} \leq \frac{\sqrt{1+4 L}-1}{2 L} \\
>0 \text { otherwise. }
\end{array}\right. \\
& -1 \leq \frac{\partial \widehat{C}}{\partial \theta_{c}}\left(\alpha^{*}\right)=-\alpha^{* L} \leq 0 \\
& 0 \leq \frac{\partial \widehat{C}}{\partial \theta_{l}}\left(\alpha^{*}\right)=\sqrt{\frac{1-\alpha^{*}}{1+\alpha^{*}}\left(1-\alpha^{* 2 L}\right)}<1 \\
& 0 \leq \frac{\partial \widehat{C}}{\partial \theta_{g}}\left(\alpha^{*}\right)=\alpha^{* L} \sqrt{\frac{1-\alpha^{*}}{1+\alpha^{*}}}<\alpha^{* L} \leq 1
\end{aligned}
$$

The comparative statics are as expected, except for $\frac{d \alpha^{*}}{d \theta_{g}}$ which reflects the counterbalancing forces that are at play when the global capacity cost increases. Increasing global capacity costs at first sight favors local sourcing. Yet, increased capacity costs also induce more smoothing. (Since the smoothing level is linked to the global sourcing allocation, this explains the counterbalancing forces. In contrast, increasing local capacity costs favors more global sourcing and more smoothing, both leading to an increase in $\alpha^{*}$.) When $\alpha=\frac{\sqrt{1+4 L}-1}{2 L}$ the global capacity cost reaches its maximum. Hence, if $\alpha<\frac{\sqrt{1+4 L}-1}{2 L}$ its marginal cost is positive. Given that an increase in $\theta_{g}$ increases marginal costs proportionally, the optimal allocation must reduce. If $\alpha^{*}>\frac{\sqrt{1+4 L}-1}{2 L}$, however, we get the opposite effect: here an increase in smoothing decreases global capacity costs because less safety capacity is needed (recall that as $\alpha \rightarrow 1$, the order policy becomes constant).

By investigating how the model parameters impact the standardized capacity costs $\theta_{l}$ and $\theta_{g}$, we find how those parameters impact the optimal smoothing level $\alpha^{*}$ and offshoring allocation $\alpha^{* L}$ :

1. If $h$ increases, then $\kappa_{I}$ increases 6 and both $\theta_{c}$ and $\theta_{l}$ decrease. If $\alpha^{*}<\frac{\sqrt{1+4 L}-1}{2 L}$, then $\theta_{g}$ increases and thus $\alpha^{*}$ decreases (less offshoring).

2. If $k^{l}$ increases while $z_{K}^{l}>0$ (which is typical), or when $z_{K}^{l}$ increases while $k^{l}>0$ (less ${ }^{6} \frac{d \kappa_{I}}{d h}=\frac{d}{d h}\left[h z_{I}+(h+b) I_{N}\left(z_{I}\right)\right]=\phi_{N}\left(z_{I}\right)+\Phi_{N}\left(z_{I}\right) z_{I}>0$ for all $z_{I}$ as optimal functions of $h$. 
flexibility in local source), then $\theta_{c}$ and $\theta_{l}$ increas $8^{7}$ and thus $\alpha^{*}$ increases (more offshoring).

3. If $k^{g}$ increases while $z_{K}^{g}>0$ (which is typical), or when $z_{K}^{g}$ increases while $k^{g}>0$ (less flexibility in global source) then $\kappa^{g}$ and $\theta_{g}$ increase while $\theta_{c}$ decreases. The overall effect depends on their relative magnitudes but typically the latter effect dominates and $\alpha^{*}$ decreases (less offshoring).

The limiting case $L \rightarrow \infty$ gives an upper bound on $C^{*}$ and insight into the benefits of smoothing:

Proposition 9 With normal demand, as $L \rightarrow \infty$, the optimal smoothing level and cost converge to that of single local sourcing smoothing:

$$
\alpha^{*} \rightarrow \alpha_{\infty}^{*}=\frac{\theta_{l}}{\theta_{l}+\left(1+L_{l}\left(1-\alpha_{\infty}^{* 2}\right)\right)^{\frac{1}{2}}},
$$

so that the optimal strategic allocation $\alpha^{* L} \simeq \alpha_{\infty}^{* L} \rightarrow 0$ as $L \rightarrow \infty$.

This means that for large leadtimes, it remains optimal to smooth at about $\alpha_{\infty}^{*}$, reflecting the lower safety capacity needs (compared to the modest safety stock increase). This limiting smoothing level $\alpha_{\infty}^{*}$ decreases as inventory costs increase relative to capacity costs. Yet, the reliance on the global source decreases exponentially. Indeed, recall that the strategic allocation is $\alpha^{L}$ and the optimal allocation decreases as $L$ increases to a small but positive level $\left(\alpha_{\infty}^{*}\right)^{L}$. This convergence is quicker when inventory costs increase relative to capacity costs (then $\alpha_{\infty}^{*}$ decreases). Theoretically, this means that a dual sourcing strategy with very small reliance on the global source remains optimal for high leadtimes. In practice, this suggests single local sourcing with order smoothing (see equation (11)).

Given that the optimal cost increases as $L$ increases, the second insight from the proposition is that dual sourcing smoothing dominates single local sourcing smoothing (and thus also single local sourcing using a base stock policy given Prop. 4). This insight is strengthened when considering only local capacity costs, as we show next.

\subsection{Local Capacity Costs $\left(\theta_{l}>0\right.$ but $\left.\theta_{g}=0\right)$}

To quantify the impact of capacity, we first consider local capacitated supply and uncapacitated global supply (i.e., $\theta_{g}=0$ ). That setting is not only more tractable but also more relevant when local capacity is less flexible than global. (Capacity in high cost countries typically is subject to significant labor regulations and strong unions, or a high degree of automation to substitute for high labor cost. Either way, such "local" capacity tends to be more expensive and inflexible than in low cost countries that feature cheap and abundant labor that yield significant capacity flexibility.) The optimal smoothing level can be specified using Lagrange's inversion series:

\footnotetext{
${ }^{7} \frac{d \kappa^{i}}{d k^{i}}=\frac{d}{d k^{i}}\left[k^{i} z_{K}^{i}+o^{i} I_{N}\left(z_{K}^{i}\right)\right]=z_{K}^{i}$ for all $z_{K}^{i}$ as optimal functions of $k^{i}$.
} 
Proposition 10 With normal demand, and if $\theta_{g}=0$, the optimal smoothing level $\alpha^{*}$ and allocation $\alpha^{* L}$ depend on $L, \theta_{c}$ and $\theta_{l}: \alpha^{*}=\frac{\theta_{l}}{1+\theta_{l}}+O\left(\left(\frac{\theta_{l}}{1+\theta_{l}}\right)^{2}\right)$ if $L \theta_{c}+\sqrt{L} \theta_{l} \leq 1$ and elsewhere

$$
\alpha^{*}=\sqrt{1-\left(L \theta_{c}+\sqrt{L} \theta_{l}\right)^{-\frac{2}{3}}+\frac{L-L_{l}-2}{3}\left(L \theta_{c}+\sqrt{L} \theta_{l}\right)^{-\frac{4}{3}}+O\left(\left(L \theta_{c}+\sqrt{L} \theta_{l}\right)^{-\frac{5}{3}}\right)}
$$

This reveals four insights: First, the key metric that drives the optimal smoothing level and offshoring allocation decision is $L \theta_{c}+\sqrt{L} \theta_{l}$, so that $\theta_{c}$ and $\theta_{l}$ are substitutes, up to a factor $\sqrt{L}$. Thus, local capacity costs have a similar impact as the standardized cost advantage. Second, the local leadtime $L_{l}$ continues to play a second order role. Third, the Lagrange formula provides again a simple approximation and bound for $\alpha^{*}$ :

Proposition 11 With normal demand, if $L \theta_{c}+\sqrt{L} \theta_{l}>1$, then the optimal smoothing level $\alpha^{*}$ has a square root approximation $\alpha_{0}$ where

$$
\alpha_{0}=\sqrt{1-\left(L \theta_{c}+\sqrt{L} \theta_{l}\right)^{-\frac{2}{3}}}
$$

that is asymptotically correct as $L \theta_{c}+\sqrt{L} \theta_{l} \rightarrow \infty$. If $L_{l}=0$, the approximation is a lower bound $\left(\alpha_{0} \leq \alpha^{*}\right)$ if $L \leq 2$, and an asymptotic upper bound otherwise.

The fourth insight from the Lagrange formulae is that dual sourcing with the DSS policy is significantly more attractive when local supply is capacitated and leadtimes increase. In contrast to uncapacitated sourcing, DSS then always dominates local single sourcing LS (and increasingly so as local capacity costs increase) and also global single sourcing (GS) using the standard base-stock policy over a parameter domain that enlarges for large leadtime differences. This finding can be corroborated and generalized analytically:

Proposition 12 With normal demand, in the presence of local capacity costs, DSS outperforms $L S$ and $G S$ if

$$
1<L \theta_{c}+\sqrt{L} \theta_{l}<\left(\frac{2}{3} \sqrt{L+1}\right)^{3}+O\left(\left(L \theta_{c}+\sqrt{L} \theta_{l}\right)^{-\frac{1}{3}}\right)
$$

and the maximal relative value $\bar{V}$ of dual sourcing over $L S\left(V=1-\widehat{C}^{*} / \widehat{C}^{l}\right)$ is an increasing value of the leadtime difference:

$$
\bar{V}=1-\frac{3}{2} \frac{(\sqrt{L}(\sqrt{L+1}-1))^{\frac{1}{3}}}{\sqrt{L+1}}+\frac{\frac{L-2}{8}(\sqrt{L}(\sqrt{L+1}-1))^{-\frac{1}{3}}}{\sqrt{L+1}}+O\left(L^{-2.5}\right) .
$$

The parameter domain (22) where DSS outperforms single sourcing essentially is a simplex that increases as the leadtime difference increases. The maximal value $(23)$ increases as the leadtime difference increases as shown in Fig. 8 and the numerical accuracy quickly improves. (E.g., when 


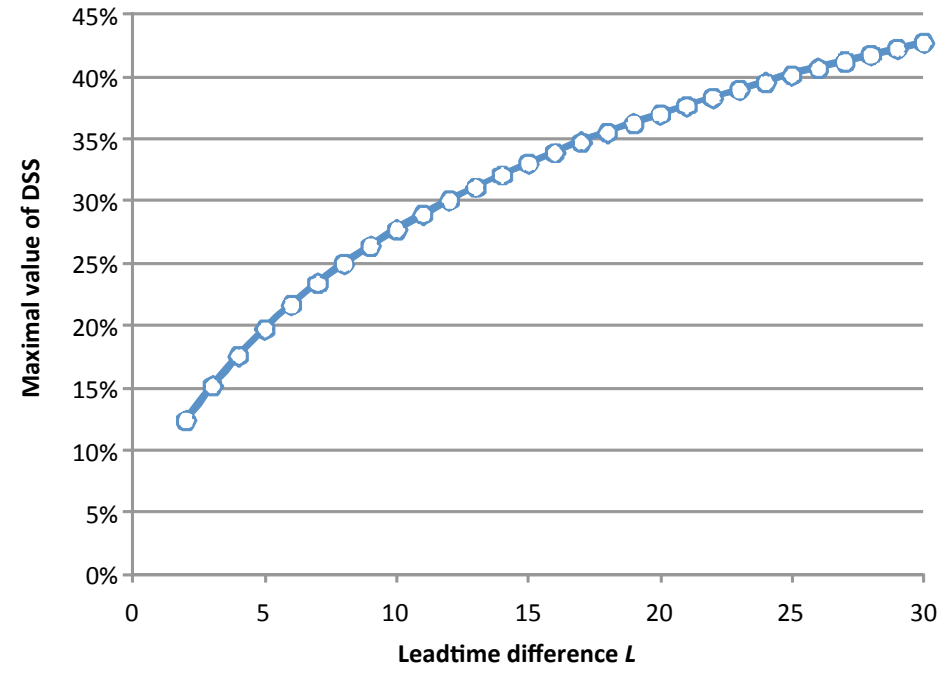

Figure 8: With local capacitated supply, the maximal relative value of DSS over LS and GS increases as the leadtime difference $L$ increases.

$L=10$, equation 23 yields $27.7 \%$ while $\bar{V}=28.4 \%$ ). The reason behind the impact of leadtime difference is that, the higher the leadtime difference between the sources, the higher the benefit of combining two sources using smoothing. The latter reduces the overall variance, and thus the capacity costs, and this reduction is larger as the leadtime difference increases.

The proposition compares DSS with single sourcing policies using a base-stock policy, but its message extends to single sourcing smoothing and the optimal Capacitated Single Sourcing (SCC) policy (discussed in section 3.4). Given that SCC cannot be optimized analytically, we numerically optimized the simulated cost under this policy and compared it to the analytically optimized cost under the DSS policy. Fig. 9 shows the magnitude of the cost reduction under DSS relative to the cost under the SCC policy. With no sourcing cost advantage $\left(\theta_{c}=0\right)$ and $L=1$, the optimal SCC policy (by definition) outperforms the DSS policy, but for moderate $\theta_{c}$, DSS performs better than SCC. As $\theta_{l}$ rises, local capacity becomes more constrained and both the gain, and the domain where DSS outperforms SCC, shrink.

\subsection{Local and Global Capacity Costs $\left(\theta_{l}>0\right.$ and $\left.\theta_{g}>0\right)$}

When both sources are capacitated, local and global capacity costs have counteracting effects on smoothing and offshoring as shown earlier in Prop. 8. For an interior, dual sourcing solution $\alpha^{*} \in(0,1)$ to exist, $\theta_{c}$ or $\theta_{l}$ must be sufficiently positive to offset a positive $\theta_{g}$. Indeed, notice that if $\theta_{g}>0$ while $\theta_{c}=\theta_{l}=0$, the cost is

$$
\widehat{C}(\alpha)=\theta_{g} \alpha^{L} \sqrt{\frac{1-\alpha}{1+\alpha}}+\sqrt{L_{l}+\frac{1}{1-\alpha^{2}}} \geq \widehat{C}(0)
$$



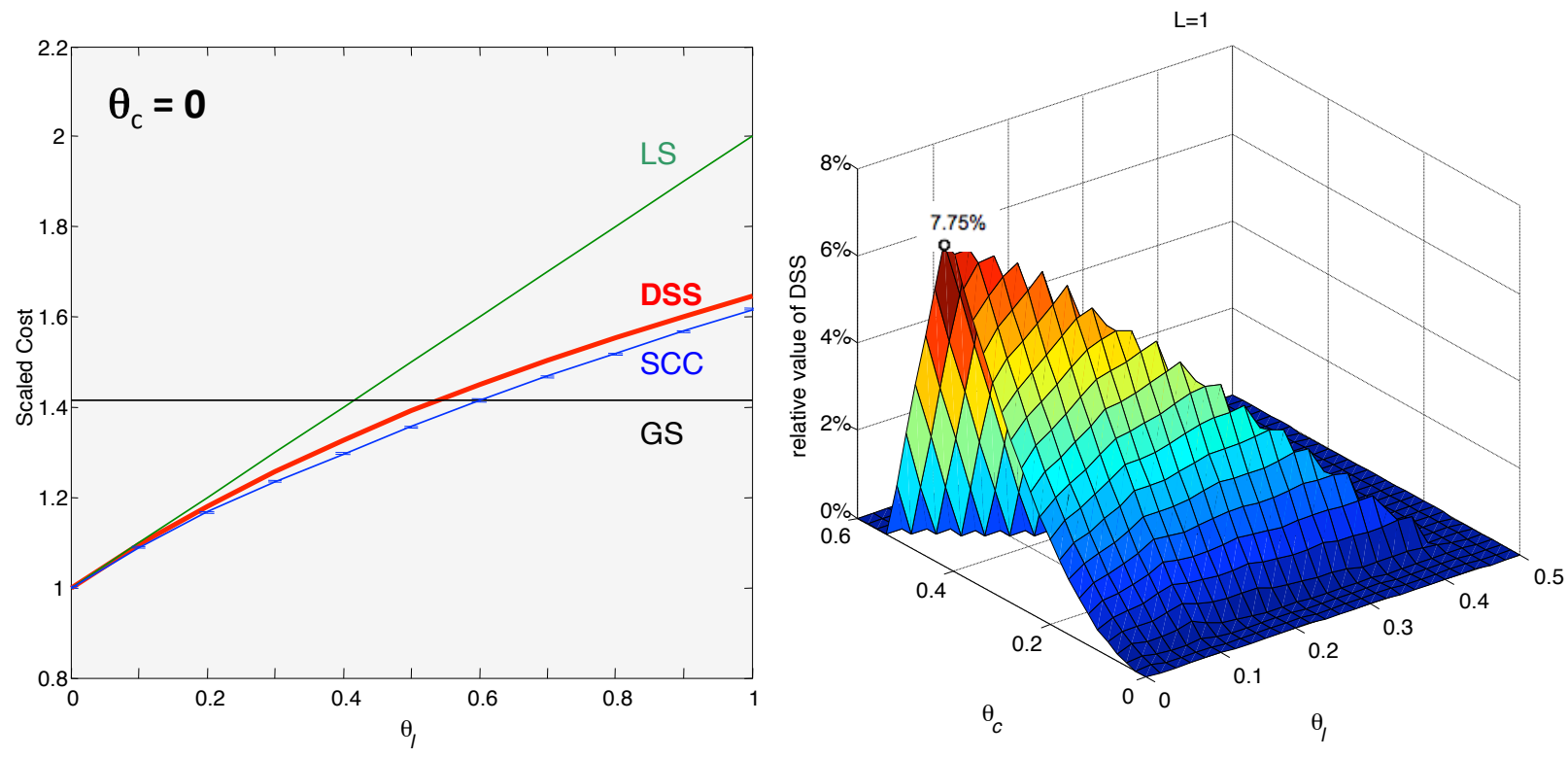

Figure 9: The relative value of Dual Sourcing Smoothing over local Single-Sourcing-Capacitated SCC (which is the optimal strategy when $\theta_{c}=0$ and $\theta_{l}<.6$, as shown in left panel). When the global source has a sourcing cost advantage (right panel), the DSS policy outperforms SCC. $\left(L=1, L_{l}=0, \theta_{g}=0\right)$

so that $\alpha^{*}=0$ and LS is optimal. When $\theta_{g}>0$ and $\theta_{c}+\theta_{l}>0$, there exist no general simple formulae to express the optimal smoothing level. (Even when $L=1$, the FOC is a 6 -th order polynomial.) Even the Langrage solutions become exceedingly complex (and we relegate them to the Technical Companion) but they do suggest that the optimal solution is a function of $L \theta_{c}+\sqrt{L} \theta_{l}$ and $\theta_{g}$. However, given that $\alpha^{*}$ decreases as $\theta_{g}$ increases (Prop. 8), the exact solutions in the previous section (only local capacity costs) provide upper bounds for the general case.

Additional results are found by approximating or bounding the marginal costs. For $L=1$, a linear approximation of the FOC around $\alpha=0$ (light offshoring) yields

$$
\widehat{M B}(\alpha)=\theta_{c}+\theta_{g}(-1+2 \alpha)+\theta_{l}+o(\alpha)=\widehat{M C}(\alpha)=\left(1+L_{l}\right)^{\frac{-1}{2}} \alpha+o(\alpha) .
$$

For $L=1$, the marginal costs are also bounded:

$$
\widehat{M B}(0)=\theta_{c}+\theta_{l}-\theta_{g}=\left(1-\underline{\alpha}_{1}^{2}\right)^{-3 / 2} \leq \widehat{M B}\left(\alpha^{*}\right)=\widehat{M C}\left(\alpha^{*}\right) \leq\left(1-\alpha^{*^{2}}\right)^{-3 / 2} .
$$

A similar bounding can be done for $L>1$, which yields:

Proposition 13 Under normal demand, the following bounds apply to the general case $\theta_{i}>0$ :

1. For $L=1$ : if $\theta_{c}+\theta_{l}-\theta_{g}>1$, then the optimal smoothing level and offshoring allocation has a lower bound

$$
\alpha^{*} \geq \underline{\alpha}_{1}=\sqrt{1-\left(\theta_{c}+\theta_{l}-\theta_{g}\right)^{-2 / 3}}
$$


that is asymptotically tight as $\theta_{c}+\theta_{l}-\theta_{g} \rightarrow \infty$. Otherwise, if $0 \leq \theta_{c}+\theta_{l}-\theta_{g}<1$ and $\theta_{g}<\frac{1}{2}$ :

$$
\alpha^{*}=\frac{\theta_{c}+\theta_{l}-\theta_{g}}{\left(1+L_{l}\right)^{\frac{-1}{2}}-2 \theta_{g}}+o\left(\frac{\theta_{c}+\theta_{l}-\theta_{g}}{\left(1+L_{l}\right)^{\frac{-1}{2}}-2 \theta_{g}}\right) \text {. }
$$

2. For any $L>1$ : if $\frac{4}{3 \sqrt{3}} \theta_{l}-\theta_{g} \frac{e^{-1 / 2}}{2} \frac{L}{\sqrt{L-1}}>1$, then the optimal smoothing level has a lower bound

$$
\alpha^{*} \geq \underline{\alpha}_{2}=\sqrt{1-\left(\frac{4}{3 \sqrt{3}} \theta_{l}-\theta_{g} \frac{e^{-1 / 2}}{2} \frac{L}{\sqrt{L-1}}\right)^{-2 / 3}}
$$

Proposition 13 presents a useful lower bound for $L=1$ that captures the three key parameters where local and global capacity costs counteract each other $\left(\theta_{l}\right.$ and $\theta_{g}$ have opposite signs). The bound (26) shows that this counteraction extends to general $L$. Unfortunately, we have not been able to generate additional insightful analytic results.

Despite the limited tractability of the general case, Proposition 13 does shine a light on recent evolutions in global labor markets. A growing number of American companies are moving their manufacturing back to the US because of higher Chinese labor costs. While workers in developing Asian countries are slowly acquiring more rights (increasing $\theta_{g}$ ), there are signs that labor in rich countries is becoming more flexible (decreasing $\theta_{l}$ ) (The Economist, Jan. 19, 2013). At the same time, The Economist reports that Europe's inflexible and costly labor markets vis-à-vis the US is one of the reasons why reshoring is largely an American phenomenon (compared to Europe). Only when national government is making the business environment attractive enough, companies will want to come back. Spurred by the Euro-crisis, some European countries have now introduced substantial labor-market reforms to remain competitive (e.g., western car workers are willing to work in night shifts again). Our results predict that this will indeed work in favor of backshoring work to the developed countries.

\section{Robustness and Comparison with Other Dual Sourcing Policies}

\subsection{Robustness for non-normal demand}

Given that our analysis assumes normally distributed demand, we want to understand how sensitive the results are to this distributional assumption. Figure 10 shows the optimal smoothing level $\alpha^{*}$ for the uncapacitated and capacitated setting when demand follows a Gamma, lognormal, (discrete) geometric and (continuous) uniform distribution, in comparison with the optimal smoothing level assuming normal demand, $\alpha_{N}^{*}$, with identical average and $C V$. The functional dependence of $\alpha^{*}$ on the standardized cost advantage or local capacity cost follows that of the normally distributed demand with identical $C V$. More importantly, the cost-difference of a mis-estimate of $\alpha^{*}$ in case of non-normal demand is minimal (see Figure 11). 

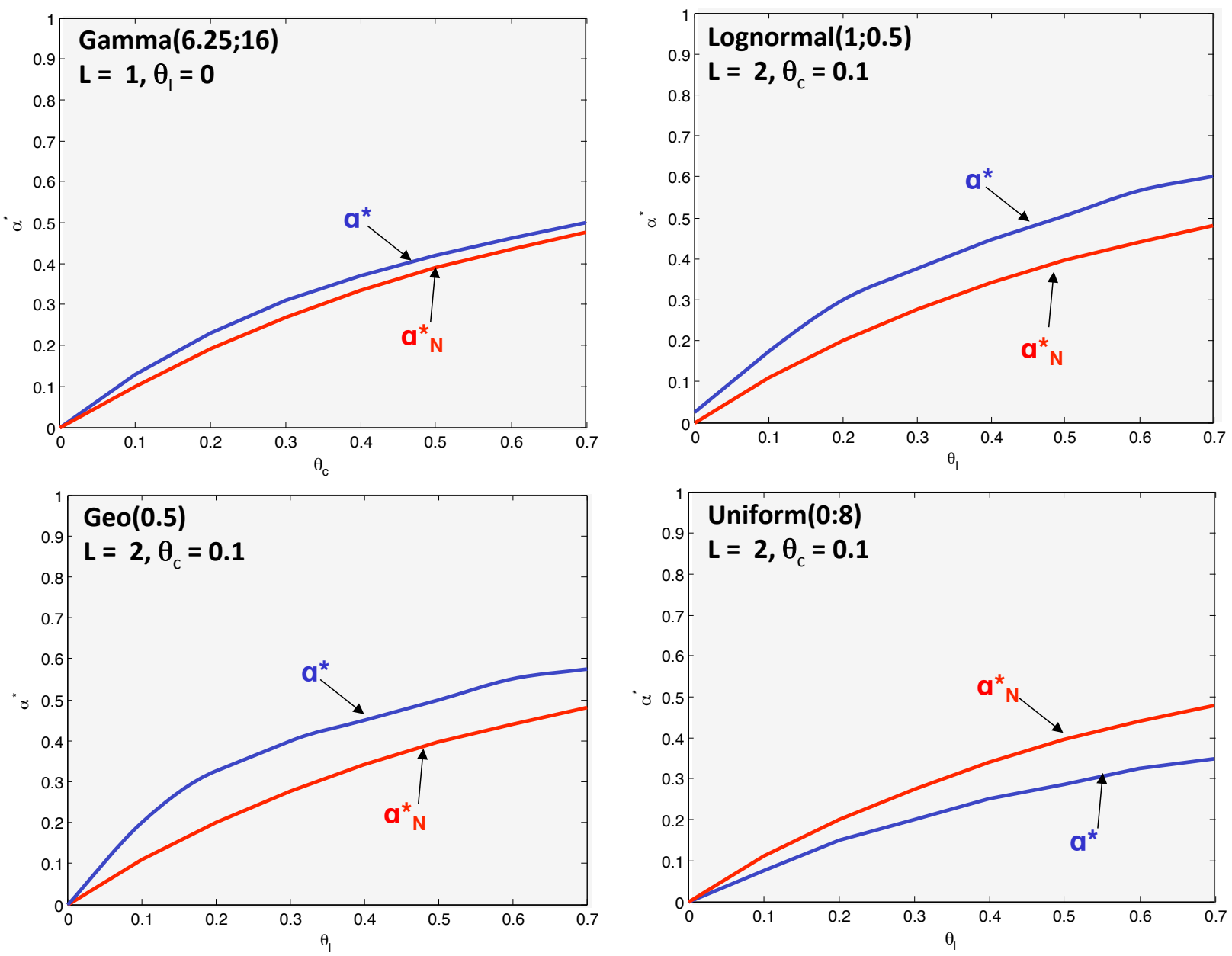

Figure 10: The optimal smoothing level $\alpha^{*}$ for non-normal demand follows the optimal smoothing level $\alpha_{N}^{*}$ assuming normally distributed demand with identical relative uncertainty $\left(\theta_{g}=0, L_{l}=0\right)$.

Of course, the distributional assumptions are less important as the variability decreases. Therefore, the reported cases not only give almost worst case comparisons, but given that the probability of negative demand exceeds $0.6 \%$ for CV exceeding 0.4 , they also are pushing the limit of the normal distribution assumption. Nevertheless, even with the assumed Geometric distribution $(C V=1.4)$ the analytic formulae (assuming normal distribution) are still remarkably good approximations. If average demand and standard deviation are scaled up in the conventional sense (as e.g. in Allon and Van Mieghem (2010)), we expect the appropriately-scaled version of our results to be asymptotically optimal. The scaled system with lead-times is however non-trivial and would be a research project in its own.

These results provide some numerical evidence that our analysis remains valid to give guidance in the strategic sourcing allocations in function of the financial parameters, in practical settings regardless of the distributional assumptions of the demand. 

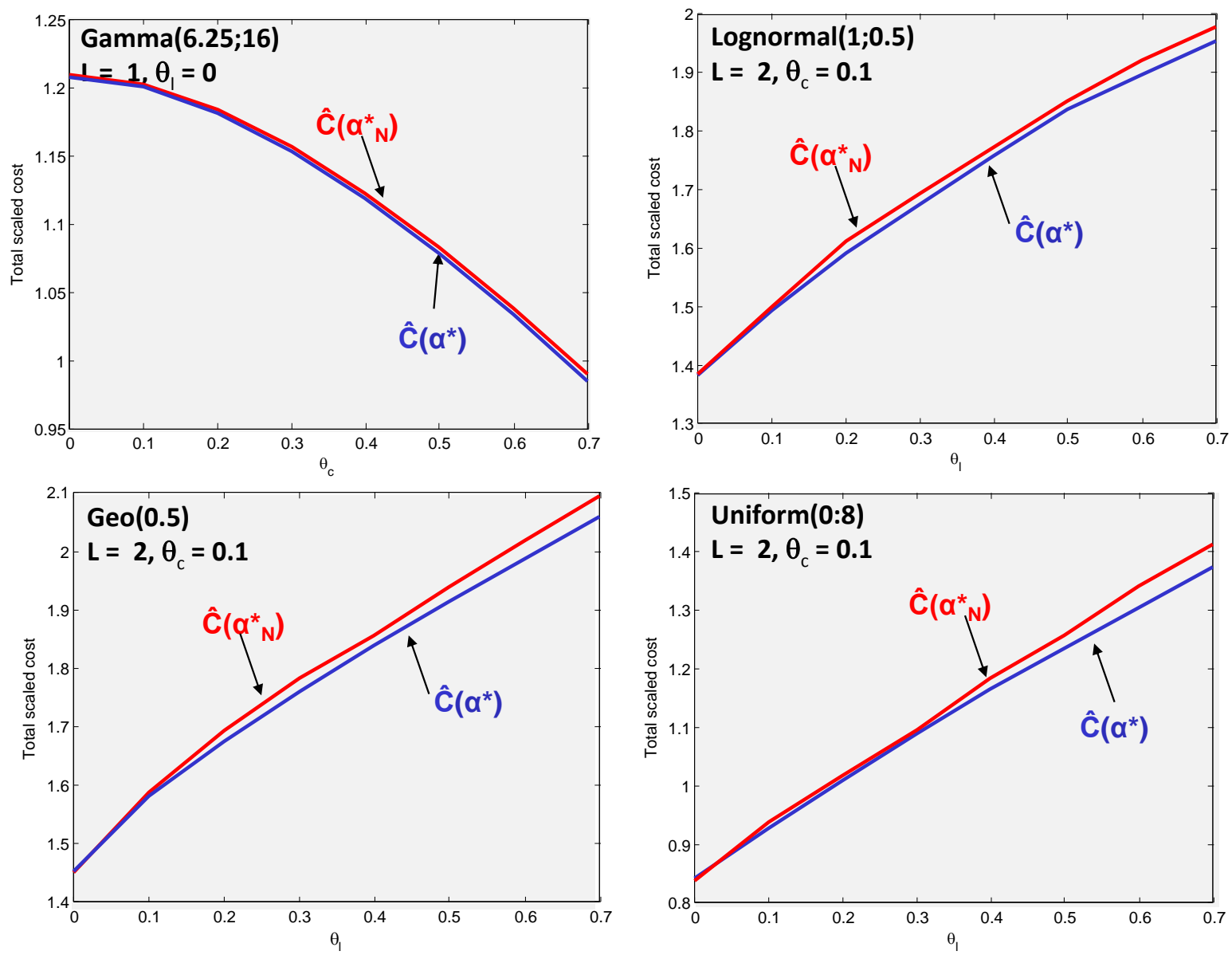

Figure 11: The total scaled cost with non-normal demand $\widehat{C}\left(\alpha^{*}\right)$ compared to the scaled cost assuming the $\alpha_{N}^{*}$ under normal demand with identical relative uncertainty $\left(\theta_{g}=0, L_{l}=0\right)$.

\subsection{Performance of DSS policy compared to other Dual Sourcing Policies}

In this section we compare the performance of the Dual Sourcing Smoothing (DSS) policy with other existing policies that have been shown to perform well in a dual sourcing setting.

The dual base-stock policies are shown to be optimal in minimizing sourcing and inventory costs for a leadtime difference $L=1$ (Fukuda 1964) and near-optimal for longer leadtime differences (Veeraraghavan and Scheller-Wolf 2008, Scheller-Wolf et al. 2006). An important distinction is the state dimension: the Single-Index policy (SI) uses one state variable, being the total inventory position, whereas the Dual-Index (DI) policy tracks two state variables, i.e. the inventory position of the local source and the total inventory position, to place resp. local and global orders. The Vector-base-stock policy (VBS) keeps track of the local inventory position and the recently placed global orders.

These policies have been shown to perform well if only sourcing and inventory costs are considered, and Veeraraghavan and Scheller-Wolf (2008), Scheller-Wolf et al. (2006) and Sheopuri et al. (2010) resp. present efficient solution procedures to find the optimal base-stock levels for the DI, SI 

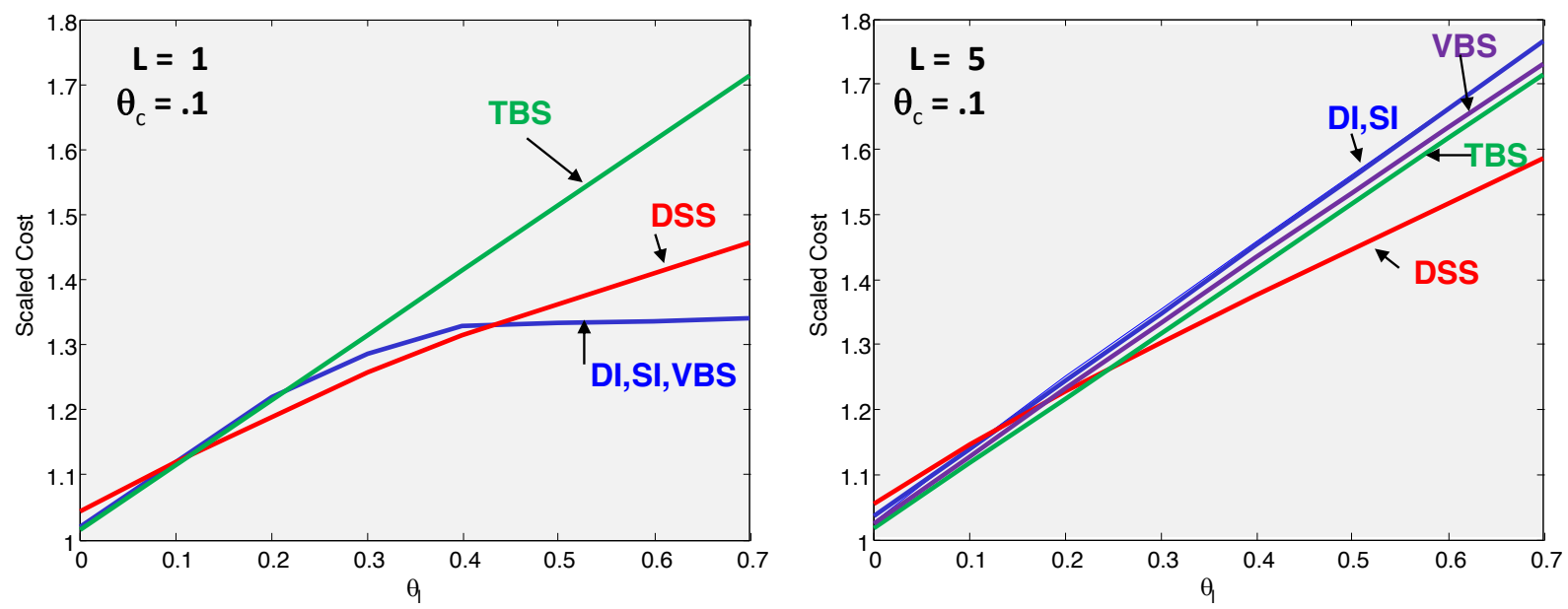

Figure 12: Scaled cost under five dual sourcing policies under normal demand $\left(\theta_{g}=0, L_{l}=0\right)$.

and VBS policies. However, they do not explicitly take into account capacity costs nor capacity flexibility. To minimize the sum of average sourcing, inventory and capacity costs per unit, as defined in (2), we therefore numerically optimized the target base-stock levels and the installed capacities at each source. There is no general closed-form distribution for the orders and net inventory in these policies (the global order may push the inventory position above its target level, causing an overshoot, so that no local order is placed), hence we resort to simulation for performance analysis and optimization.

We also benchmark with the standing order, or Tailored Base-Surge (TBS) policy, which is as well a base-stock type policy: the global source supplies every period a constant rate $q^{g}<\mu$, and an additional order to the local source is placed only when the inventory falls below the target level. In the TBS policy, the standing order may exceed the observed demand, resulting in excess inventory excursions above its target level. In essence, the "excess inventory process" behaves like a regulated random walk and the safety stock increases as the standing order to the global source goes up (since the exponential tail of the regulated random walk will be longer as $q^{g}$ increases). The TBS policy requires the evaluation of a $G / D / 1$ queue, where the inter-arrival time has the per period demand distribution and the processing time is the fixed order size from the global source. For certain choices of the demand distribution, this is a tractable problem (as shown by Janakiraman et al. (2014)), but for general demand distributions we need to rely on a simulation analysis to optimize the target base-stock and capacity levels.

We conducted an extensive simulation study to analyze the impact of capacity and leadtime differences in the uncapacitated and local capacitated setting $\left(\theta_{g}=0\right.$ and $\left.L_{l}=0\right)$. Fig. 12 shows representative findings which can be summarized as follows:

(a) Without capacity costs $\left(\theta_{l}=\theta_{g}=0\right)$, DSS is outperformed by the other dual sourcing policies. This is to be expected given that dual base-stock policies are optimal in minimizing sourcing and inventory costs when $L=1$, and near-optimal for longer leadtime differences. Their perfor- 

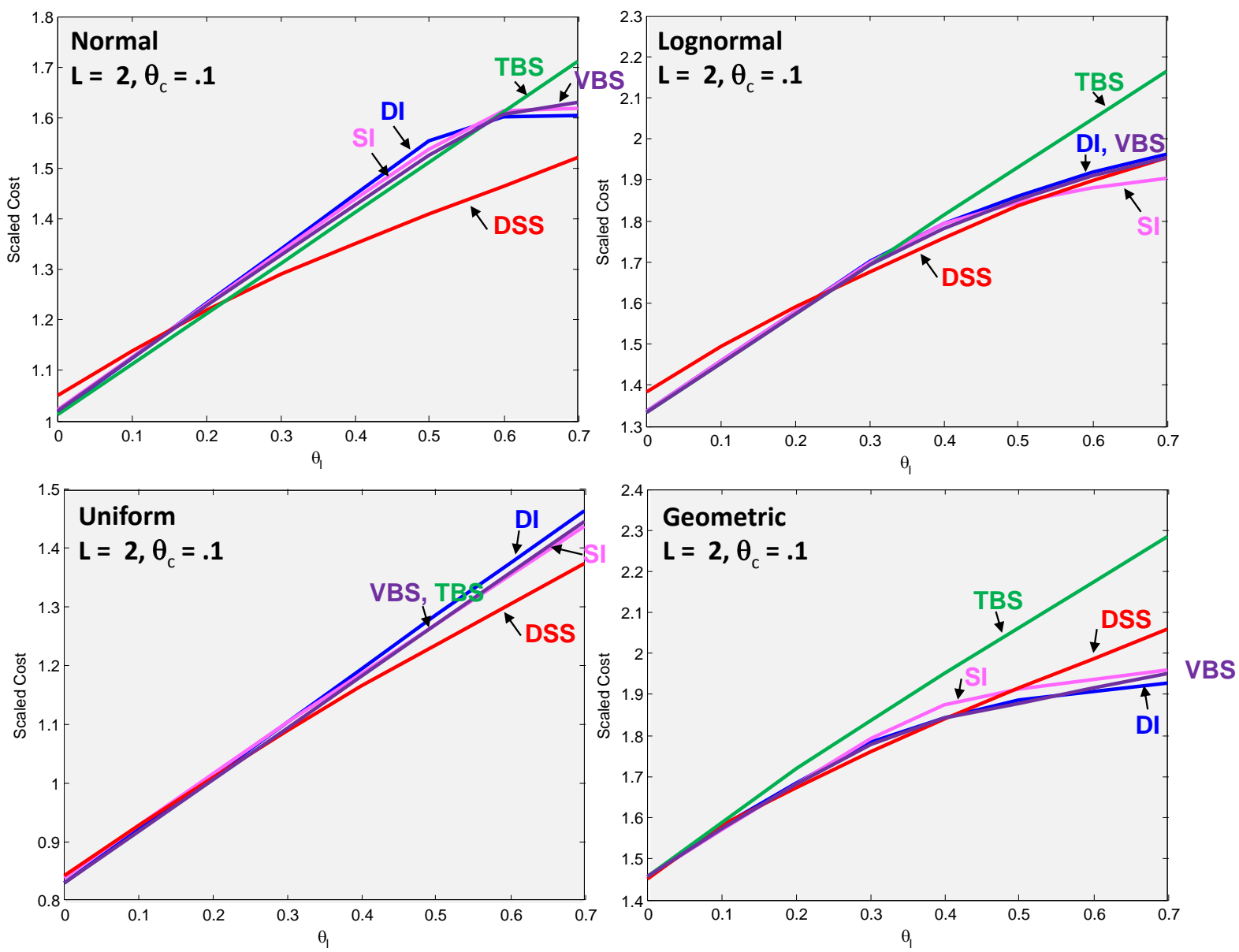

Figure 13: Scaled cost under five dual sourcing policies under normal and non-normal demand $\left(\theta_{g}=0, L_{l}=\right.$ $0)$.

mance superiority derives from their ability to react promptly to demand fluctuations. However, DSS is smoothing out the demand fluctuations in its orders (see Eq. (9P)); TBS is constrained by its standing global order but has dynamic local orders. Due to its smoothing behavior, DSS (and to a lesser extent TBS) needs more safety stock to act as a buffer between volatile demand and smooth replenishments, leading to increased inventory holding costs compared to the dual base-stock policies. The TBS policy improves as the leadtime difference increases (as also shown by Janakiraman et al. (2014)).

(b) With capacity costs, DSS can outperform the dual base-stock policies because smoothing reduces both local and global order variability and thus safety capacity requirements and costs. (Note that TBS completely smoothens (levels) global orders and requires no global but more local safety capacity; the dual base-stock policies do not smooth at all and hence require higher local and global safety capacity). This reduction in capacity costs may compensate the increased inventory holding costs of smoothing. DSS outperforms the other policies when capacity costs are dominating: the higher the sourcing cost advantage $\theta_{c}$, the more the capacity $\operatorname{cost} \theta_{l}$ should dominate for DSS 
to outperform the other policies. At the same time, the dual base-stock policies converge naturally to global single sourcing when $\theta_{l}$ increases, which may in turn outperform DSS (DSS does not naturally converge to single global sourcing).

(c) DSS performs best for longer leadtime differences $L$ : DSS rapidly outperforms the other policies, even for low values of the capacity $\operatorname{cost} \theta_{l}$ : in that case, the reduced capacity requirements quickly compensate for the (relatively modest) increase in safety stocks due to order smoothing.

These findings are valid under both normal and non-normal demand. Figure 13 illustrates the performance of the DSS policy compared to these reference policies under a lognormal, (continuous) uniform, (discrete) geometric, and normal demand distribution. These distributions are inspired by the above mentioned reference papers in the dual sourcing literature, that use the same distributions in their numerical experiments (Veeraraghavan and Scheller-Wolf 2008, Scheller-Wolf et al. 2006, Sheopuri et al. 2010).

In summary, DSS is a reasonable policy that is typically competitive to the reference dual sourcing policies, and can perform better in the presence of high capacity costs and high leadtime differences.

\section{Summary and Discussion}

The main contribution of this paper is the inclusion of capacity cost and flexibility in a strategic global sourcing decision, in addition to considering sourcing cost and leadtimes (as in the existing literature). We propose a linear control policy that performs well in both a single sourcing and dual sourcing setting. An attractive feature of our analysis is the identification and analysis of three dimensionless parameters that, in addition to the leadtimes, are the four key forces behind

the sourcing decision: $\theta_{c}$ (which captures the financial trade-off, the inventory service level, the volatility in demand) and $\theta_{l}$ and $\theta_{g}$ (which contain the ratio of capacity cost vs. holding cost of resp. the local and global source, and their flexibility to work in overtime). These data are typically available inside the firm (although one may have to search for them in different departments), and we are now able to quantify how they contribute to strategic sourcing allocations decisions based on these data. These parameters also allow the comparison of different firms with different scales.

A numerical study shows that our analytic results for normally distributed demand are robust for other distributions and that the smoothing policy is competitive to existing single and dual sourcing policies in terms of total cost minimization. The study also confirms expectations that the smoothing policy performs better in the presence of high capacity costs and high leadtime differences given that it explicitly takes the capacity costs and leadtime difference into account.

As with any analytic study, ours begs for extensions. The assumption of normally distributed demand is essential in deriving all the analytical results: Direct extensions could study demand distributions with higher coefficients of variation (e.g., between 1 and 2, or even up to 3 Muckstadt 1997)), or with "fat tails," which we expect to favor more local sourcing, or with serial demand correlation, where negative correlation will favor smoothing and global sourcing. Another extension could analyze non-stationary demand patterns to model for example how the sourcing allocation 
should adapt to the product life cycle $\square^{8}$ A third extension could insert unit cost or quantity uncertainty to model for example currency exchange rate risk or supply quality risk. Last, but not least, would be a game-theoretic multi-decision maker model where one firm (the buyer) sources from one or two independent firms. These non-obvious model extensions are full research projects by themselves.

Acknowledgments: The authors are indebted to Piet Van Mieghem for introducing us to the Lagrange inversion theorem and to the anonymous reviewers for their improvement suggestions.

\section{Appendix: Lagrange's Inversion Theorem and Proof of Equation (19)}

Markushevich (1985, II, pp. 88) gives Lagrange's series for the inverse of a complex function $f(z)$ that is analytic around $z_{0}$ with $f^{\prime}\left(z_{0}\right) \neq 0$ as:

$$
f^{-1}(z)=z_{0}+\left.\sum_{n=1}^{\infty} \frac{1}{n !}\left[\frac{d^{n-1}}{d z^{n-1}}\left(\frac{z-z_{0}}{f(z)-f\left(z_{0}\right)}\right)^{n}\right]\right|_{z=z_{0}}\left(z-f\left(z_{0}\right)\right)^{n} .
$$

If $f^{(m)}\left(z_{0}\right)=0$ but $f^{(k)}\left(z_{0}\right) \neq 0$ for all $m<k$, then 27) generalizes to Markushevich 1985, II, pp. 92)

$$
f^{-1}(z)=z_{0}+\left.\sum_{n=1}^{\infty} \frac{1}{n !}\left[\frac{d^{n-1}}{d z^{n-1}}\left(\frac{z-z_{0}}{\left[f(z)-f\left(z_{0}\right)\right]_{s}^{1 / k}}\right)^{n}\right]\right|_{z=z_{0}}\left(z-f\left(z_{0}\right)\right)^{n / k}
$$

where the subscript $s$ denotes any fixed single-valued branch of the $k$-valued function $\left[f(z)-f\left(z_{0}\right)\right]^{1 / k}$.

If $L_{l}=0$, consider the function $f(z)=z^{3}(1-z)^{m}$ which is analytic in $z$ because $m=L-2 \geq 0$ is integer. Given that $f^{\prime}(0)=f^{\prime \prime}(0)=0$ but $f^{(3)}(0)=3 !>0,28$ yields around $z_{0}=0$ :

$$
\left.\frac{d^{j}}{d z^{j}}(1-z)^{-n \frac{m}{3}}\right|_{z=0}=\left.\frac{\Gamma\left(\frac{n m}{3}+j\right)}{\Gamma\left(\frac{n m}{3}\right)}(1-z)^{-\frac{n m}{3}-j}\right|_{z=0}=\frac{\Gamma\left(\frac{n m}{3}+j\right)}{\Gamma\left(\frac{n m}{3}\right)},
$$

and we obtain the Lagrange series for the inverse function,

$$
f^{-1}(z)=\sum_{n=1}^{\infty} \frac{\Gamma\left(n\left(\frac{m}{3}+1\right)-1\right)}{\Gamma\left(\frac{n m}{3}\right)} \frac{z^{n / 3}}{n !}
$$

where $m=L-2$. (The Technical Companion shows that the radius of convergence is exactly $\theta \geq \underline{\theta}_{L}$.) Here we were able to calculate each term in the series explicitly due to the simple form of $f$. If $L_{l}>0$ the derivation becomes more complex but is still doable in closed form; with capacity costs, however, the derivation is term by term and cumbersome, as shown in the Technical Companion.

\footnotetext{
${ }^{8}$ One could apply our formula for each phase separately to predict that the sourcing allocation will move from emphasizing local during product introduction (low mean but high variability in demand) to offshore during maturity and back to local during decline. However, this remains an unverified extrapolation of a stationary result.
} 


\section{References}

Allon, G., J. A. Van Mieghem. 2010. Global dual sourcing. Management Science 56(1) 110-124.

Balakrishnan, A., J. Geunes, M. S. Pangburn. 2004. Coordinating supply chains by controlling upstream variability propagation. Manufacturing \& Service Operations Management 6(2) 163-183.

Bertrand, J.W.M. 1986. Balancing production level variations and inventory variations in complex production systems. International Journal of Production Research 24(5) 1059-1074.

Bray, R., H. Mendelson. 2012. Information transmission and the bullwhip effect: an empirical investigation. Management Science 58(5) 860-875.

Cachon, G.P., T. Randall, G.M. Schmidt. 2007. In search of the bullhwip effect. Manufacturing \& Service Operations Management 9(4) 457-479.

Cantor, D.E., E. Katok. 2012. Production smoothing in a serial supply chain: a laboratory investigation. Transportation Research Part E: Logistics and Transportation Review 48(4) 781-794.

Chen, L., H. Lee. 2012. Bullwhip effect measurement and its implications. Operations Research 60(4) $771-784$.

Combes, François. 2011. Inventory theory and mode choice in freight transport: The case of the simultaneous use of two transport modes on one shipper-receiver relationship. Working Paper, Université Paris Est and Association for European Transport and Contributors.

Cui, R., G. Allon, A. Bassamboo, J. A. Van Mieghem. 2014. Information sharing in supply chains: An empirical and theoretical valuation. Working paper, Kellogg School of Management, Northwestern University.

Forrester, J. 1961. Industrial Dynamics. MIT Press, Cambridge MA.

Fukuda, Y. 1964. Optimal policies for the inventory problem with negotiable lead time. Management Science 10(4) 690-708.

Galois, Évariste. 1846. Oeuvres mathématiques d'Évariste Galois. Journal des Mathématiques Pures et Appliquées (11) 381-444.

George, K., Ramaswamy S., Rassey L. 2014. Next-shoring: a ceo's guide. McKinsey Quarterly .

Goel, A., G. Gutierrez. 2011. Multi-echelon procurement and distribution policies for traded commodities. Management Science 57(12) 2228-2244.

Graves, S. C. 1988. Safety stocks in manufacturing systems. Journal of Manufacturing and Operations Management 1(1) 67-101.

Jain, N., K. Girotra, Netessine S. 2014. Managing global sourcing: an empirical study. Management Science Forthcoming.

Janakiraman, G., S. Seshadri, A. Sheopuri. 2014. Analysis of tailored base-surge policies in dual sourcing inventory systems. Management Science Forthcoming.

Lagrange, Joseph-Louis. 1770. Nouvelle méthode pour résoudre les équations littérales par le moyen des séries. Mémoires de l’Académie Royale des Sciences et Belles-Lettres de Berlin (24) 251-326.

Liu, Z., A. Nagurney. 2011. Supply chain networks with global outsourcing and quick-response production under demand and cost uncertainty. Working Paper, University of Massachusetts, Massachusetts.

Magee, J. F. 1958. Production Planning and Inventory Control. McGraw-Hill, New York.

Markushevich, A. I. 1985. Theory of functions of a complex variable, vol. 1-3. Chelsea Publishing Company, N. Y. 
Muckstadt, J.A. 1997. A paradigm lost. Technical report No. 1180, School of Operations Research and Industrial Engineering, Cornell University, Ithaca, New York.

Rosenshine, M., D. Obee. 1976. Analysis of a standing order inventory system with emergency orders. Operations Research 24(6) 1143-1155.

Scheller-Wolf, A., S. Veeraraghavan, G.J. van Houtum. 2006. Effective dual sourcing with a single index policy. Working Paper, Tepper School of Business, Carnegie Mellon University, Pittsburgh.

Sheopuri, A., G. Janakiraman, S. Seshadri. 2010. New policies for the stochastic inventory control problem with two supply sources. Operations Research 58(3) 734-745.

Simon, H. 1952. On the application of servomechanism theory in the study of production control. Econometrica 20 247-268.

Sobel, M. 1969. Production smoothing with stochastic demand I: finite horizon case. Management Science 16(3) 195-207.

Sobel, M. 1971. Production smoothing with stochastic demand II: infinite horizon case. Management Science 17(11) $724-735$.

Tagaras, G., D. Vlachos. 2001. A periodic review inventory system with emergency replenishments. Management Science 47(3) 415-429.

The Economist. 2013. Special report on outsourcing and offshoring: Here, there and everywhere. Jan. 19 URL http://www.economist.com/sites/default/files/20130119_offshoring_davos.pdf.

Vassian, H. J. 1955. Application of discrete variable servo theory to inventory control. Operations Research $3272-282$.

Veeraraghavan, S. K., A. Scheller-Wolf. 2008. Now or later: a simple policy for effective dual sourcing in capacitated systems. Operations Research 56(4) 850-864.

Whittemore, A.S., S. C. Saunders. 1977. Optimal inventory under stochastic demand with two supply options. SIAM Journal on Applied Mathematics 32(2) 293-305.

Wu, X., F. Zhang. 2011. Efficient supplier or responsive supplier? an analysis of sourcing strategies under competition. Working Paper, Olin Business School, Washington University, St. Louis.

Zipkin, P. 2008. Old and new methods for lost-sales inventory systems. Operations Research 56(5) 1256-1263.

Zipkin, P. H. 2000. Foundations of Inventory Management. McGraw-Hill, New York. 